Review

\title{
Unified Approach to Fractional Calculus Images of Special Functions-A Survey
}

\author{
Virginia Kiryakova \\ Institute of Mathematics and Informatics, Bulgarian Academy of Sciences, 1113 Sofia, Bulgaria; \\ virginia@diogenes.bg
}

Received: 17 November 2020; Accepted: 14 December 2020; Published: 21 December 2020

\begin{abstract}
Evaluation of images of special functions under operators of fractional calculus has become a hot topic with hundreds of recently published papers. These are growing daily and we are able to comment here only on a few of them, including also some of the latest of 2019-2020, just for the purpose of illustrating our unified approach. Many authors are producing a flood of results for various operators of fractional order integration and differentiation and their generalizations of different special (and elementary) functions. This effect is natural because there are great varieties of special functions, respectively, of operators of (classical and generalized) fractional calculus, and thus, their combinations amount to a large number. As examples, we mentioned only two such operators from thousands of results found by a Google search. Most of the mentioned works use the same formal and standard procedures. Furthermore, in such results, often the originals and the images are special functions of different kinds, or the images are not recognized as known special functions, and thus are not easy to use. In this survey we present a unified approach to fulfill the mentioned task at once in a general setting and in a well visible form: for the operators of generalized fractional calculus (including also the classical operators of fractional calculus); and for all generalized hypergeometric functions such as ${ }_{p} \Psi_{q}$ and ${ }_{p} F_{q}$, Fox $H$ - and Meijer $G$-functions, thus incorporating wide classes of special functions. In this way, a great part of the results in the mentioned publications are well predicted and appear as very special cases of ours. The proposed general scheme is based on a few basic classical results (from the Bateman Project and works by Askey, Lavoie-Osler-Tremblay, etc.) combined with ideas and developments from more than 30 years of author's research, and reflected in the cited recent works. The main idea is as follows: From one side, the operators considered by other authors are cases of generalized fractional calculus and so, are shown to be ( $m$-times) compositions of weighted Riemann-Lioville, i.e., Erdélyi-Kober operators. On the other side, from each generalized hypergeometric function ${ }_{p} \Psi_{q}$ or ${ }_{p} F_{q}(p \leq q$ or $p=q+1$ ) we can reach, from the final number of applications of such operators, one of the simplest cases where the classical results are known, for example: to ${ }_{0} F_{q-p}$ (hyper-Bessel functions, in particular trigonometric functions of order $\left.(q-p)\right)_{0} F_{0}$ (exponential function), or ${ }_{1} F_{0}$ (beta-distribution of form $\left.(1-z)^{\alpha} z^{\beta}\right)$. The final result, written explicitly, is that any GFC operator (of multiplicity $m \geq 1$ ) transforms a generalized hypergeometric function into the same kind of special function with indices $p$ and $q$ increased by $m$.
\end{abstract}

Keywords: fractional calculus operators; special functions; generalized hypergeometric functions; integral transforms of special functions

MSC: 26A33; 33C60; 33E12; 44A20

\section{Introduction}

Special functions (SF) have always been unavoidable tools for mathematicians, physicists, astronomers, applied scientists and engineers while looking to express and study (theoretically, in tables 
or by numerical algorithms) the solutions of treated mathematical models. On the other side, recently there has been an increased interest in fractional calculus (FC) and its applications, as evidence for which we refer the readers to the data in the survey by Machado-Kiryakova [1]. Fractional calculus is nowadays a favorite, and even a sort of fashionable research area, although the boom of publications and attempts to "fractalize" any kinds of integer order models can bring some threats to the prestige of this discipline, especially in cases of weak or wrong results and not adequate innovations. Let us mention also the phenomenon of hundreds of papers of the last few years (only a few of them can be cited here) dealing with "evaluation of FC images of SF", most of which use the same standard techniques with changing only the particular special function (SF) and the particular case of the FC operator. Furthermore, it often happens that in such results the originals and the images are special functions of different kinds, or the images are not recognized as known special functions, and thus are not easy to use. In recent papers, such as [2-5], we share our criticism on this practice and show that all such results can be derived at once by following a general approach, based on ideas from older author's works on generalized fractional calculus (GFC), since [6].

Here we try to collect the ideas, results and examples from our recent works on the subject. The survey starts with Preliminaries (Section 2) providing a short background on the considered SF and FC operators; followed by Section 3 with results for images of the generalized hypergeometric functions ${ }_{p} F_{q}$ and ${ }_{p} \Psi_{q}$ and their simpler cases under the operators the classical FC operators (Riemann-Liouville and Erdélyi-Kober integrals and derivatives of fractional order). Then, in Section 4 we present our unified approach for evaluation of GFC operators of arbitrary generalized hypergeometric functions ${ }_{p} F_{q}$ and ${ }_{p} \Psi_{q}$ ), resulting in the main Theorems 3 and 4 . This allows to handle very wide classes of operators of generalized ( $m$-tuple, $m \geq 1$ ) fractional integration and differentiation and of considered special functions. In Sections 5-7 we consider specifications of these results for the Erdélyi-Kober, Saigo and Marichev-Saigo-Maeda (M-S-M) operators, that appear as cases of our GFC, resp. for $m=1$, $m=2, m=3$, give their images for the Wright generalized hypergeometric functions, and many illustrative examples for particular results by other authors. Section 8 considers more general cases of GFC operators with arbitrary multiplicity $m \geq 1$, as the multiple Gel'fond-Leontiev operators related to the multi-index Mittag-Leffler functions, and the hyper-Bessel operators related to the hyper-Bessel functions of Delerue. In Section 9 we comment on works of other authors on introducing some "new" special functions and show that these are again Wright generalized hypergeometric functions ${ }_{p} \Psi_{q}$. Therefore, the various FC images they propose come as simple corollaries of our general results. To show the effectiveness of the proposed unified approach, in this survey we collected some 21 examples for FC images of SF, and referred to a long list of other authors' works on the subject. Section 10 summarizes some conclusions.

\section{Preliminaries}

Here we provide a short and only necessary background on the considered classes of special functions (SF) and of operators of classical FC and of generalized fractional calculus (GFC), so as to explain the general ideas. All details on defining the single-valued branches of the considered functions, functional spaces, and necessary conditions on appearing parameters, can be found in our previous works, as cited, and for example in ([6], Section 5.5.i). Basically, we consider functions in the complex plane of the form $\left\{f(z)=z^{\mu} \widetilde{f}(z), \mu \geq 0, \widetilde{f}(z)\right.$ analytic and single valued in $\left.\Omega\right\}$, where $\Omega$ is a starlike domain with respect to $z=0$, usually a disk $\Delta_{R}:|z|<R$. Most of the considered special functions are entire functions, or analytic ones in disks in $\mathbb{C}$.

The results we consider are for the classes of so-called generalized hypergeometric functions (g.h.f) with Mellin-Barnes type integral representations, namely the Fox $H$-function, Meijer $G$-function and their most widely used cases of Wright g.h.f. $p \Psi_{q}$ and g.h.f. $p F_{q}$. Even if our aim is to incorporate as large as possible classes of special functions, let us mention that other transcendental functions as the elliptic integrals, Lambert W-, Mathiew-, Zeta-, etc. functions are outside of our studies. Also, we emphasize on results for LHS integrals, although for the RHS ones similar techniques and 
results are applied; and consider Riemann-Liouville type fractional derivatives. For the Caputo-type differentiation operators, similar but different results will be exposed in a separate work.

\subsection{Special Functions of Fractional Calculus}

Under "classical" Special Functions (SF) we mean these "mathematical functions" and orthogonal polynomials of which the origin goes back to 18th and 19th centuries and are named after great mathematicians like Euler, Gauss, Riemann, Bessel, Kummer, Legendre, Laguerre. These "Special Functions of Mathematical Physics" appeared with the needs of applied sciences and serve as solutions of integer order (most commonly 2nd order) differential equations from models in mathematical physics. In the last two centuries it was observed that modeling of many phenomena of the physical and social world can be reflected much more adequately by means of differential equations of arbitrary fractional or higher integer orders, and the so-called special functions of fractional calculus (SF of FC) as providing tools for their explicit solutions became unavoidable tools in the hands of theoretical and applied scientists recognizing the power of fractional calculus (FC).

Recently, many handbooks and surveys appeared as dedicated not only to classical SF but also to the SF of FC, to mention some of them: Prudnikov-Brychkov-Marichev [7], Marichev [8], Srivastava-Gupta-Goyal [9], Kilbas-Srivastava-Trujillo [10], Podlubny [11], Kiryakova [6], Yakubovich-Luchko [12], Mathai-Haubold [13], Gorenflo-Kilbas-Mainardi-Rogosin [14]. Such a list cannot be full here, and for more sources see also the survey paper Machado-Kiryakova [1]. In the papers on the topic and in this survey, we limit ourselves to the Fox $H$-functions of one complex variable, as enough of a general level to expose the proposed approach.

Definition 1 (Ch. Fox 1960). see books such as [6,7,9,10], and earlier and latest ones) The Fox H-function is a generalized hypergeometric function, defined by means of the Mellin-Barnes type contour integral

$$
H_{p, q}^{m, n}\left[z \mid \begin{array}{c}
\left(a_{i}, A_{i}\right)_{1}^{p} \\
\left(b_{j}, B_{j}\right)_{1}^{q}
\end{array}\right]=\frac{1}{2 \pi i} \int_{\mathcal{L}} \mathcal{H}_{p, q}^{m, n}(s) z^{-s} d s, \text { with } \mathcal{H}_{p, q}^{m, n}(s)=\frac{\prod_{j=1}^{m} \Gamma\left(b_{j}+B_{j} s\right) \prod_{i=1}^{n} \Gamma\left(1-a_{i}-A_{i} s\right)}{\prod_{j=m+1}^{q} \Gamma\left(1-b_{j}-B_{j} s\right) \prod_{i=n+1}^{p} \Gamma\left(a_{i}+A_{i} s\right)},
$$

$z \neq 0$, where $\mathcal{L}$ is a suitable contour (of three possible types in $\mathbb{C}: \mathcal{L}_{-\infty}, \mathcal{L}_{\infty},(\gamma-i \infty, \gamma+i \infty)$ ), the orders $(m, n, p, q)$ are non negative integers so that $0 \leq m \leq q, 0 \leq n \leq p$, the parameters $A_{i}>0, B_{j}>0$ are positive, and $a_{i}, b_{j}, i=1, \ldots, p ; j=1, \ldots, q$ can be arbitrary complex such that $A_{i}\left(b_{j}+l\right) \neq B_{j}\left(a_{i}-l^{\prime}-1\right), l, l^{\prime}=$ $0,1,2, \ldots ; i=1, \ldots, n ; j=1, \ldots, m$. Note that the integrand $\mathcal{H}_{p, q}^{m, n}(s)$ with $s \mapsto-s$ is the Mellin transform of the H-function (1).

The details on the properties of the Fox H-function can be found in many contemporary handbooks on SF such as $[7,9,10]$, where its behavior is described in term of the denotations:

$$
\begin{gathered}
\rho=\prod_{i=1}^{p} A_{i}^{-A_{i}} \prod_{j=1}^{q} B_{j}^{B_{j}} ; \Delta=\sum_{k=1}^{j} B_{j}-\sum_{i=1}^{p} A_{i} ; \\
\mu=\sum_{j=1}^{q} b_{j}-\sum_{i=1}^{p} a_{i}+\frac{p-q}{2} ; a^{*}=\sum_{i=1}^{n} A_{i}-\sum_{i=n+1}^{p} A_{i}+\sum_{j=1}^{m} B_{j}-\sum_{j=m+1}^{q} B_{j} .
\end{gathered}
$$

Note that the H-function is an analytic function of $z$ in circle domains $|z|<\rho$ or outside them (or in sectors of them, or in the whole $\mathbb{C}$ ), depending on the above parameters and the contours. 
If all $A_{i}=B_{j}=1, i=1, \ldots, p ; j=1, \ldots, q$, the $H$-function $H_{p, q}^{m, n}\left[\begin{array}{c}z \\ \left(a_{i}, 1\right)_{1}^{p} \\ \left(b_{j}, 1\right)_{1}^{q}\end{array}\right]$ reduces to the Meijer's G-function (C.S. Meijer (1936), see details in ([15], Vol.1) and all above-mentioned books)

$$
G_{p, q}^{m, n}\left[z \mid \begin{array}{l}
\left(a_{i}\right)_{1}^{p} \\
\left(b_{j}\right)_{1}^{q}
\end{array}\right]=\frac{1}{2 \pi i} \int_{\mathcal{L}} \mathcal{G}_{p, q}^{m, n}(s) z^{-s} d s=\frac{1}{2 \pi i} \int_{\mathcal{L}} \frac{\prod_{j=1}^{m} \Gamma\left(b_{j}+s\right) \prod_{i=1}^{n} \Gamma\left(1-a_{i}-s\right)}{\prod_{j=m+1}^{q} \Gamma\left(1-b_{j}-s\right) \prod_{i=n+1}^{p} \Gamma\left(a_{i}+s\right)} z^{-s} d s, \quad z \neq 0 .
$$

Although simpler than (1), the G-function is yet enough general as it incorporates the Classical SF (known also as Named SF) and many elementary functions. See lists of examples, for example, in ([15], Vol.1), ([6], Appendix C).

Now, we attract the readers' attention to the most typical examples of SF of FC, which are Fox $H$-functions but not reducible to Meijer $G$-functions in the general case (of irrational $A_{j}, B_{k}$ ). These originate from works of Sir Edward Maitland (E.-M.) Wright in a series of his works (1935-1940).

Definition 2 (see, e.g., $\left([6,7,14]\right.$, App.E)). The Wright generalized hypergeometric function ${ }_{p} \Psi_{q}(z)$, called also Fox-Wright function (F-W g.h.f.) is defined as:

$$
\begin{gathered}
p_{p} \Psi_{q}\left[\begin{array}{c}
\left(a_{1}, A_{1}\right), \ldots,\left(a_{p}, A_{p}\right) \\
\left(b_{1}, B_{1}\right), \ldots,\left(b_{q}, B_{q}\right)
\end{array} \mid z\right]=\sum_{k=0}^{\infty} \frac{\Gamma\left(a_{1}+k A_{1}\right) \ldots \Gamma\left(a_{p}+k A_{p}\right)}{\Gamma\left(b_{1}+k B_{1}\right) \ldots \Gamma\left(b_{q}+k B_{q}\right)} \frac{z^{k}}{k !} \\
=H_{p, q+1}^{1, p}\left[\begin{array}{c|c}
-z & \left(1-a_{1}, A_{1}\right), \ldots,\left(1-a_{p}, A_{p}\right) \\
(0,1),\left(1-b_{1}, B_{1}\right), \ldots,\left(1-b_{q}, B_{q}\right)
\end{array}\right] .
\end{gathered}
$$

In terms of parameters (2), the ${ }_{p} \Psi_{q}$-function is an entire function of $z$ if $\Delta>-1$, while for $\Delta=-1$, it is an absolutely convergent series in the disk $\{|z|<\rho\}$, and also for $|z|=\rho$ if $\operatorname{Re}(\mu)>1 / 2$, see, for example, [16].

If all $A_{1}=\cdots=A_{p}=1, B_{1}=\cdots=B_{q}=1$, the Wright g.h.f. reduces to the generalized hypergeometric ${ }_{p} F_{q}$-function, which is a case of the $G$-function (3), see details in ([15], Vol.1):

$$
\begin{aligned}
& { }_{p} \Psi_{q}\left[\begin{array}{c}
\left(a_{1}, 1\right), \ldots,\left(a_{p}, 1\right) \\
\left(b_{1}, 1\right), \ldots,\left(b_{q}, 1\right)
\end{array} \mid z\right]=c_{p} F_{q}\left(a_{1}, \ldots, a_{p} ; b_{1}, \ldots, b_{q} ; z\right)=\sum_{k=0}^{\infty} \frac{\left(a_{1}\right)_{k} \ldots\left(a_{p}\right)_{k}}{\left(b_{1}\right)_{k} \ldots\left(b_{q}\right)_{k}} \frac{z^{k}}{k !} \\
& =G_{p, q+1}^{1, p}\left[-z \mid \begin{array}{c}
1-a_{1}, \ldots, 1-a_{p} \\
0,1-b_{1}, \ldots, 1-b_{q}
\end{array}\right] ; \quad \text { where } c=\left[\prod_{i=1}^{p} \Gamma\left(a_{i}\right) / \prod_{j=1}^{q} \Gamma\left(b_{j}\right)\right],(a)_{k}:=\Gamma(a+k) / \Gamma(a) .
\end{aligned}
$$

The Mittag-Leffler (M-L) function, introduced by G. Mittag-Leffler (1902-1905), with extended 2-parameters' definition by R.P. Agarwal (1953), was presented yet in Bateman Project's [15], Vol.3 (1954), in a chapter for "Miscellaneous Functions". However, it was ignored for a long time in the books on special functions because the applied scientists suffered from a lack of tables for its Laplace transforms. Although appearing from studies not related to fractional calculus, nowadays the M-L function has become the most popular and most exploited SF of FC, honored to be the "Queen"-function of FC. See details, for example, in [14], also in $[6,17,18]$.

Definition 3. The Mittag-Leffler (M-L) functions $E_{\alpha}$ and $E_{\alpha, \beta}$, are entire functions of order $\rho=1 / \alpha$ and type 1 , defined by the power series

$$
E_{\alpha}(z)=\sum_{k=0}^{\infty} \frac{z^{k}}{\Gamma(\alpha k+1)}, E_{\alpha, \beta}(z)=\sum_{k=0}^{\infty} \frac{z^{k}}{\Gamma(\alpha k+\beta)}, \quad \alpha>0, \beta>0 .
$$


As "fractional index" $(\alpha>0)$ analogs of the exponential and trigonometric functions that satisfy ODEs of 1st and 2nd order $(\alpha=1,2)$, the M-L functions serve as solutions of fractional order differential equations. A M-L type function with three indices, known as the Prabhakar function (T.R. Prabhakar, 1971 ) is also often studied and used, for details see [14,17-19], and other contemporary books and surveys on M-L type functions:

$$
E_{\alpha, \beta}^{\gamma}(z)=\sum_{k=0}^{\infty} \frac{(\gamma)_{k}}{\Gamma(\alpha k+\beta)} \frac{z^{k}}{k !}, \quad \alpha, \beta, \gamma \in \mathbb{C}, \operatorname{Re} \alpha>0,
$$

where $(\gamma)_{0}=1,(\gamma)_{k}=\Gamma(\gamma+k) / \Gamma(\gamma)$ denotes the Pochhammer symbol. For $\gamma=1$ we get the M-L function $E_{\alpha, \beta}$, and if additionally $\beta=1$, it is $E_{\alpha}$.

These M-L type functions are simple cases of the Wright g.h.f. and of the $H$-function, namely:

$$
\begin{gathered}
E_{\alpha, \beta}(z)={ }_{1} \Psi_{1}\left[\begin{array}{r}
(1,1) \\
(\beta, \alpha)
\end{array} \mid z\right]=H_{1,2}^{1,1}\left[-z \mid \begin{array}{c}
(0,1) \\
(0,1),(1-\beta, \alpha)
\end{array}\right], \\
E_{\alpha, \beta}^{\gamma}(z)=\frac{1}{\Gamma(\gamma)}{ }_{1} \Psi_{1}\left[\begin{array}{r}
(\gamma, 1) \\
(\beta, \alpha)
\end{array} \mid z\right]=H_{1,2}^{1,1}\left[\begin{array}{c}
(1-\gamma, 1) \\
(0,1),(1-\beta, \alpha)
\end{array}\right] .
\end{gathered}
$$

A vector index extension of (7) appeared in the works by Luchko et al. (for example, Yakubovich-Luchko [12]) on operational calculus' methods for some fractional order PDE. Under the name multi-index (multiple) M-L function, it was introduced by Kiryakova [20] using a different approach, via the Gelfond-Leontiev generalized integration and differentiation operators (see Section 8). Further, these functions are studied in detail by Kiryakova [21,22], by Kilbas-Koroleva-Rogosin [23], Paneva-Konovska [19], and many other followers.

Definition 4 (Kiryakova [21,22]). Let $m>1$ be an integer, $\left(\alpha_{1}>0, \alpha_{2}>0, \ldots, \alpha_{m}>0\right)$ and $\left(\beta_{1}, \beta_{2}, \ldots, \beta_{m}\right)$ be arbitrary real parameters. By means of these "multi-indices", the multi-index Mittag-Leffler function (multi-M-Lf.) is the entire function defined as:

$$
\begin{aligned}
& E_{\left(\alpha_{i}\right),\left(\beta_{i}\right)}(z):=E_{\left(\alpha_{i}\right),\left(\beta_{i}\right)}^{(m)}(z)=\sum_{k=0}^{\infty} \frac{z^{k}}{\Gamma\left(\alpha_{1} k+\beta_{1}\right) \ldots \Gamma\left(\alpha_{m} k+\beta_{m}\right)} \\
& ={ }_{1} \Psi_{m}\left[\begin{array}{c}
(1,1) \\
\left(\beta_{i}, \alpha_{i}\right)_{1}^{m}
\end{array} \mid z\right]=H_{1, m+1}^{1,1}\left[-z \mid \begin{array}{c}
(0,1) \\
(0,1),\left(1-\beta_{i}, \alpha_{i}\right)_{1}^{m}
\end{array}\right] .
\end{aligned}
$$

Under weakened restrictions on the $\alpha$ 's not obligatory to be all nonnegative, the study was extended by Kilbas et al; see Kilbas-Koroleva-Rogosin [23].

The basic properties and results for the functions (9) and long lists of their examples, all of them having wide applications in solutions of integer- and fractional-order models, are provided in our previous papers like Kiryakova [21,22,24]. Let us shortly mention particular cases like: for $m=1$, we have the classical M-L function $E_{\alpha, \beta}$ with all its particulars (error-, incomplete gamma-, Rabotnov, etc., 
functions); and for $m>1$ (many of them treated in the examples in next sections) (see ([15], Vol.2, Section 7.5.4, Section 7.5.5), [25]):

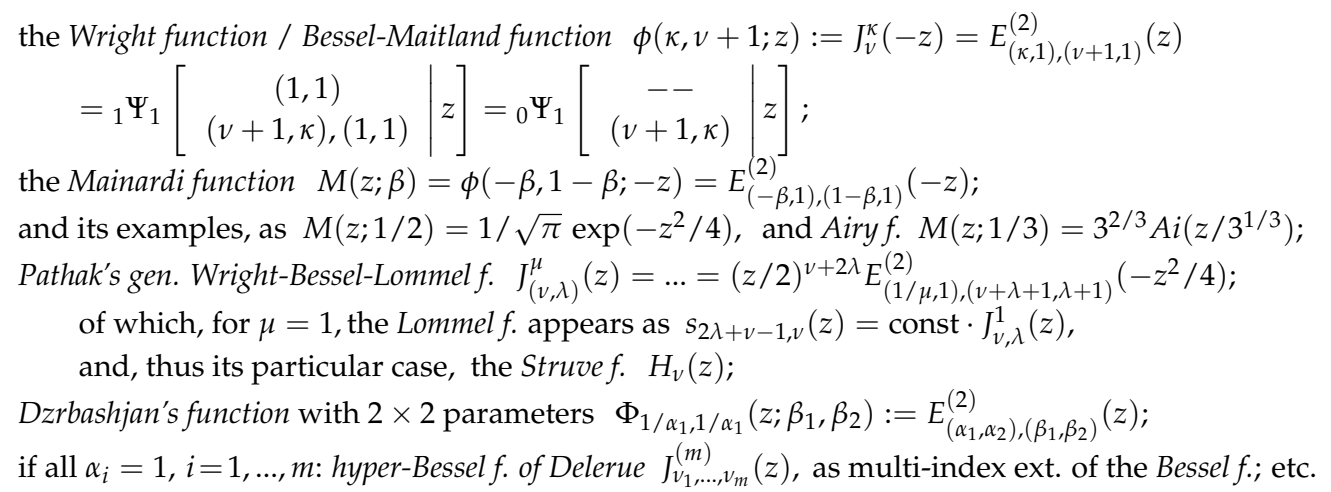

Recently, in Kilbas-Koroleva-Rogosin [23] the definition (9) has been extended for arbitrary values of the $\alpha$ 's parameters. Paneva-Konovska introduced and studied generalizations of the Prabhakar function (8) by means of three sets of parameters $\left(\alpha_{1}>0, \alpha_{2}>0, \ldots, \alpha_{m}>0\right),\left(\beta_{1}, \beta_{2}, \ldots, \beta_{m}\right)$, $\left(\gamma_{1}, \gamma_{2}, \ldots, \gamma_{m}\right)$, called 3m-parametric $M$-L functions, see, for example, $[19,26]$ and references therein. Multivariate and matrix extensions of the M-L and multi-index M-L functions are also explored.

In another survey paper, Kiryakova [27], we are exposing many other details on the theory of the SF of FC, in the sense of Wright generalized hypergeometric functions ${ }_{p} \Psi_{q}$ and multi-index Mittag-Leffler functions, and provide an extensive list of their particular cases, studied in theoretical and applicable aspects by various authors.

Remark 1. The techniques of the Mellin transform

$$
\mathfrak{M}\{f(z) ; s\}:=F^{*}(s)=\int_{0}^{\infty} f(z) z^{s-1} d t
$$

is one of the main tools to evaluate integrals and various integral transforms of special functions, including their images under operators of FC. After some classical publications of previous centuries, the main contribution to this approach is due to Marichev [8]. He proposed a natural but wide ranged scheme, based on the contour integral representations of Mellin-Barnes type for the $H$ - and G-functions, like (1) and (3). Note that the integrands $\mathcal{H}_{p, q}^{m, n}$ and $\mathcal{G}_{p, q}^{m, n}$ are their Mellin transforms (of variable $s \mapsto-s$ ) are fractions of products of $2 \times 2$ groups of Gamma-functions, and each special function being a special case of the generalized hypergeometric functions, has a particular representation of that kind. For example ([10], (1.11.24)):

$$
\mathfrak{M}\left\{{ }_{p} \Psi_{q}\left[\begin{array}{c}
\left(a_{i}, A_{i}\right)_{1}^{p} \\
\left(b_{j}, B_{j}\right)_{1}^{a}
\end{array} \mid-z\right] ; s\right\}=\frac{\Gamma(s) \prod_{i=1}^{p} \Gamma\left(a_{i}-s A_{i}\right)}{\prod_{j=1}^{q} \Gamma\left(b_{j}-s B_{j}\right)} .
$$

For variations of results, one can use in addition the relations (see, e.g., in ([28], (2.6)-(2.8))):

$\mathfrak{M}\{f(\lambda z) ; s\}=\lambda^{-s} F^{*}(s+\gamma), \lambda>0 ; \mathfrak{M}\left\{z^{\gamma} f(z) ; s\right\}=F^{*}(s+\gamma) ; \mathfrak{M}\left\{f\left(z^{\mu}\right) ; s\right\}=\frac{1}{\mu} F^{*}\left(\frac{s}{\mu}\right), \mu>0$

Examples for the use of the Mellin transform in this respect are given (among many others works) in: Luchko and Kiryakova ([28], Section 4) (general scheme and examples with the M-L and Wright functions), Agarwal, Rogosin, and Trujillo [29] and Paneva-Konovska and Kiryakova [30] (images for multi-index $M-L$ functions and their particular cases). 


\subsection{Operators of Generalized Fractional Calculus}

In fractional calculus (FC), meant as a theory of the integration and differentiation of arbitrary (including fractional, not obligatorily integer) order, there are several almost equivalent definitions for "fractional" integrals and derivatives, applied in various functional spaces. Here we are interested in evaluating FC operator images of special functions, defined by power series, most of which are entire functions, or at least analytic ones inside/outside disks in a complex plane. Therefore, we restrict our statements to such functions, although they hold also for spaces of weighted continuous or Lebesgue integrable functions on the real half-line.

For the basic background on FC theory and related topics as SF, integral transforms, generalizations and applications, we can refer to the books (among many others, for a longer list see, e.g., Machado and Kiryakova [1]), such as those by: Samko, Kilbas, and Marichev [31], Podlubny [11], Kilbas, Srivastava, and Trujillo [10], Yakubovich and Luchko [12], including the author's one, Kiryakova [6] and a recent one, Sandev and Tomovski [32].

We state results for the Riemann-Liouville (R-L) operator for integration $R^{\delta}$ of order $\delta>0$, the corresponding R-L fractional derivative $D^{\delta}$, and its counterpart ${ }^{*} D^{\delta}$ in the Caputo sense, that is only the left-hand sided operators of FC (and skip similar details for the Weyl-type, right-hand sided operators).

The main operator of fractional integration we consider is the Erdélyi-Kober operator (E-K) of integration of order $\delta>0$, depending on two additional parameters $\gamma \in \mathbb{R}$ and $\beta>0$,

$$
I_{\beta}^{\gamma, \delta} f(z)=\frac{1}{\Gamma(\delta)} \int_{0}^{1} \sigma^{\gamma}(1-\sigma)^{\delta-1} f\left(z \sigma^{\frac{1}{\beta}}\right) d \sigma=\frac{z^{-\beta(\gamma+\delta)}}{\Gamma(\delta)} \int_{0}^{z}\left(z^{\beta}-\xi^{\beta}\right)^{\delta-1} \xi^{\beta \gamma} f(\xi) d\left(\xi^{\beta}\right),
$$

note it is the identity for $\delta=0$. Especially for functional spaces of weighted analytic functions of the form $f(z)=z^{\mu} \widetilde{f}(z), \mu \geq 0$ (see beginning of Section 2), to be preserved by this operator, we require $\gamma>-1-\frac{\mu}{\beta}$, in addition to $\delta \geq 0, \beta>0$. This operator, more general than the R-L integral, and having many more applications, was introduced in Sneddon's works, such as [33], and is considered in books $([6,10,31]$, Ch.2), and recently in many other works on fractional order models. The Erdélyi-Kober-type fractional integrals, or briefly Erdélyi-Kober integrals, of the form

$$
I f(z)=z^{\delta_{0}} I_{\beta}^{\gamma, \delta} f(z), \text { with } \delta_{0} \geq 0 .
$$

are basic in our studies, and are called classical fractional integrals, and we consider their commutable compositions that are presented as our generalized fractional integrals, Kiryakova [6,34].

The Erdélyi-Kober operator (13) reduces to the R-L operator of integration for $\gamma=0, \beta=1, \delta_{0}=\delta$,

$$
R_{0+, z}^{\delta} f(z):=R^{\delta} f(z)=z^{\delta} I_{1}^{0, \delta} f(z) ; \text { and conversely, } I_{1}^{\gamma, \delta} f(z)=z^{-\gamma-\delta} R^{\delta} z^{\gamma} f(z) .
$$

Note that some authors often refer to the Erdélyi-Kober integral (12) as Euler integral transformation, when they are to handle various integral transforms of special functions.

The fractional order derivative of R-L type corresponding to the E-K integral (12), called E-K fractional derivative $D_{\beta}^{\gamma, \delta}$, is an extension of the R-L fractional derivative $D^{\delta} f(z):=\left(\frac{d}{d z}\right)^{n} R^{n-\delta} f(z)$. Instead of $(d / d z)^{n}$, a suitably chosen auxiliary differential operator $D_{n}$ of integer order is used, a polynomial of the Euler differential operator $(z d / d z)$. It has been introduced and studied in the works of Kiryakova and Luchko et al., ([6], Ch.2) and ([12], Ch.3) and in the next ones, as [35],

$$
D_{\beta}^{\gamma, \delta} f(z)=D_{n} I_{\beta}^{\gamma+\delta, n-\delta} f(z)=\prod_{j=1}^{n}\left(\frac{1}{\beta} z \frac{d}{d z}+\gamma+j\right) I_{\beta}^{\gamma+\delta, n-\delta} f(z), \quad n-1<\delta \leq n, n \in \mathbb{N} .
$$


The more formal representation ([6], Ch.1, Equation (1.6.7))

$$
D_{\beta}^{\gamma, \delta} f(z)=\left[z^{-\gamma} D^{\delta} z^{\gamma+\delta} f\left(z^{1 / \beta}\right)\right]_{z \mapsto z^{\beta}}
$$

serves to provide a better understanding on the structure and nature of (15).

The Caputo-type analogs of the R-L and E-K fractional derivatives are defined in the same way but with exchanged order of the nonnegative order integration and the integer order differentiation, see, for example, [36], also [35], namely,

$$
{ }^{*} D_{\beta}^{\gamma, \delta} f(z)=I_{\beta}^{\gamma+\delta, n-\delta} D_{n} f(z) .
$$

The notion of generalized fractional integration operators was introduced by S. Kalla (1969-1979), who suggested the common form of such operators (see details and references in [37]),

$$
I f(z)=\int_{0}^{1} \Phi(\sigma) \sigma^{\gamma} f(z \sigma) d \sigma=z^{-\gamma-1} \int_{0}^{z} \Phi\left(\frac{\xi}{z}\right) \xi^{\gamma} f(\xi) d \xi,
$$

where $\Phi(\sigma)$ is an arbitrary continuous (analytical) function for which the integral makes sense, most commonly a special function as the Bessel, Gauss, G- or $H$-function. The operators of such generalized fractional calculus (GFC) are expected to include, in particular, these of the classical FC and should satisfy the main axioms for the FC theory.

Note that for a rather general or rather narrow choice of the special function $\Phi$, only some formal operational rules for the generalized fractional integrals (17) can be provided. Therefore, in our generalized fractional calculus (GFC), Kiryakova [6], the suitable choice of the kernel-functions $\Phi$ as $G_{m, m}^{m, 0}$ and $H_{m, m}^{m, 0}$-functions was crucial. In that case, the generalized fractional integrals can be decomposed into commutative products of operators of classical FC (Erdélyi-Kober operators). Thus, the tools of the special functions and the wide usage of the classical FC are combined into a GFC with developed detailed theory and many established applications.

Definition 5 (Kiryakova [6]). The multiple E-K integral (of multiplicity $m>1$ ), is defined by means of the real parameters' sets $\left(\delta_{1} \geq 0, \ldots, \delta_{m} \geq 0\right)$-multi-order of integration, $\left(\gamma_{1}, \ldots, \gamma_{m}\right)$ - multi-weight; and $\left(\beta_{1}>0, \ldots, \beta_{m}>0\right)$-additional multi-parameter, as:

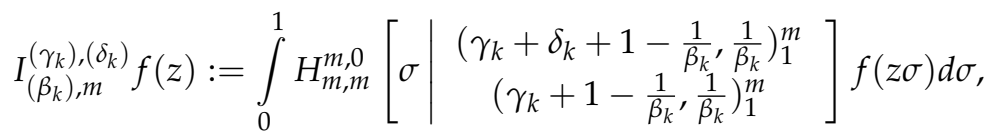

if $\sum_{k=1}^{m} \delta_{k}>0$; and as the identity operator: $I_{\left(\beta_{k}\right), m}^{\left(\gamma_{k}\right),(0, \ldots, 0)} f(z)=f(z)$, if $\delta_{1}=\delta_{2}=\cdots=\delta_{m}=0$.

Note that the above kernel $H_{m, m}^{m, 0}$-function is an analytic function in the unit disk and $H_{m, m}^{m, 0}(\sigma) \equiv 0$ for $|\sigma|>1$ (Kiryakova, [6]). Specially for functional spaces of weighted analytic functions of the form $f(z)=z^{\mu} \widetilde{f}(z), \mu \geq 0$ (see beginning of Section 2), to be preserved by this operator, we require $\gamma_{k}>-1-\frac{\mu}{\beta_{k}}$, in addition to $\delta_{k} \geq 0, \beta_{k}>0$.

If all the $\beta^{\prime}$ s are equal: $\beta_{1}=\beta_{2}=\ldots=\beta_{m}=\beta>0$, then (18) has a simpler representation where the kernel is a $G_{m, m}^{m, 0}$-function of Meijer, which is also analytic in unit disk and $G_{m, m}^{m, 0}(\sigma) \equiv 0$ for $|\sigma|>1$,

$$
I_{(\beta, \ldots, \beta), m}^{\left(\gamma_{k}\right),\left(\delta_{k}\right)} f(z):=I_{\beta, m}^{\left(\gamma_{k}\right),\left(\delta_{k}\right)} f(z)=\int_{0}^{1} G_{m, m}^{m, 0}\left[\begin{array}{c}
\left(\gamma_{k}+\delta_{k}\right)_{1}^{m} \\
\left(\gamma_{k}\right)_{1}^{m}
\end{array}\right] f\left(z \sigma^{1 / \beta}\right) d \sigma=\left[\prod_{k=1}^{m} I_{\beta}^{\gamma_{k}, \delta_{k}}\right] f(z) .
$$


The operators of the form

$$
\widetilde{I} f(z)=z^{\delta_{0}} I_{\left(\beta_{k}\right), m}^{\left(\gamma_{k}\right),\left(\delta_{k}\right)} f(z), \quad \widetilde{I} f(z)=z^{\delta_{0}} I_{\beta, m}^{\left(\gamma_{k}\right),\left(\beta_{k}\right)} f(z), \text { with } \delta_{0} \geq 0,
$$

are both referred to shortly as generalized fractional integrals of multi-order $\left(\delta_{1}, \ldots, \delta_{m}\right)$.

The following decomposition property is proved in [6], etc. (see, e.g., decomposition Th.5.2.1 in [6]). It is important because the GFC integrals (18) and (19) can be represented not only by using the kernel Fox $H$ - and $G$-functions, but also by means of the repeated integral representations for the commutative product of classical E-K operators (12):

$$
I_{\left(\beta_{k}\right), m}^{\left(\gamma_{k}\right),\left(\delta_{k}\right)} f(z):=\left[\prod_{k=1}^{m} I_{\beta_{k}}^{\gamma_{k}, \delta_{k}}\right] f(z)=\int_{0}^{1} \ldots \int_{0}^{1}\left[\prod_{k=1}^{m} \frac{\left(1-\sigma_{k}\right)^{\delta_{k}-1} \sigma_{k}^{\gamma_{k}}}{\Gamma\left(\delta_{k}\right)}\right] f\left(z \sigma_{1}^{1 / \beta_{1}} \ldots \sigma_{m}^{1 / \beta_{m}}\right) d \sigma_{1} \ldots d \sigma_{m} .
$$

In the book Kiryakova [6] and subsequent papers, we have provided the operational properties of the operators (18) and (19) as semigroup property, formal inversion formula, reduction to identity or to the conventional integration operators for special parameters' choice. This is to justify their names as operators of GFC.

Following the idea of how the R-L and E-K fractional derivatives are defined, we have proposed the definition of the corresponding generalized fractional derivatives. To this end, the auxiliary differential operator $D_{\eta}$, a polynomial of $z\left(\frac{d}{d z}\right)$ of degree $\eta_{1}+\ldots+\eta_{m}$, is used:

$$
D_{\eta}=\left[\prod_{r=1}^{m} \prod_{j=1}^{\eta_{r}}\left(\frac{1}{\beta_{r}} z \frac{d}{d z}+\gamma_{r}+j\right)\right], \quad \text { with } \eta_{k}:=\left\{\begin{array}{ll}
{\left[\delta_{k}\right]+1,} & \text { for noninteger } \delta_{k}, \\
\delta_{k}, & \text { for integer } \delta_{k},
\end{array} \quad k=1, \ldots, m .\right.
$$

Definition 6 (Kiryakova ([6], Ch.1,Ch.5), [34,35]). The multiple (m-tuple) Erdélyi-Kober fractional derivative of $R$ - $L$ type of multi-order $\delta=\left(\delta_{1} \geq 0, \ldots, \delta_{m} \geq 0\right)$ is defined by means of the differ-integral operator:

$$
D_{\left(\beta_{k}\right), m}^{\left(\gamma_{k}\right),\left(\delta_{k}\right)} f(z):=D_{\eta} I_{\left(\beta_{k}\right), m}^{\left(\gamma_{k}+\delta_{k}\right),\left(\eta_{k}-\delta_{k}\right)} f(z)=D_{\eta} \int_{0}^{1} H_{m, m}^{m, 0}\left[\begin{array}{c}
\sigma \\
\left(\gamma_{k}+\eta_{k}+1-\frac{1}{\beta_{k}}, \frac{1}{\beta_{k}}\right)_{1}^{m} \\
\left(\gamma_{k}+1-\frac{1}{\beta_{k}}, \frac{1}{\beta_{k}}\right)_{1}^{m}
\end{array}\right] f(z \sigma) d \sigma .
$$

Analogously, the Caputo-type generalized fractional derivative has been introduced in Kiryakova and Luchko [35], as

$$
{ }^{*} D_{\left(\beta_{k}\right), m}^{\left(\gamma_{k}\right),\left(\delta_{k}\right)} f(z)=I_{\left(\beta_{k}\right), m}^{\left(\gamma_{k}+\delta_{k}\right),\left(\eta_{k}-\delta_{k}\right)} D_{\eta} f(z) .
$$

For all equal $\beta^{\prime}$ s: $\beta_{1}=\ldots=\beta_{m}=\beta>0$, the R-L and Caputo-type "derivatives" corresponding to the generalized fractional integral (19) has a simpler form with Meijer G-function in the kernel:

$$
\begin{gathered}
D_{\beta, m}^{\left(\gamma_{k}\right),\left(\delta_{k}\right)} f(z)=D_{\eta} I_{\beta, m}^{\left(\gamma_{k}+\delta_{k}\right),\left(\eta_{k}-\delta_{k}\right)} f(z)=\left[\prod_{r=1}^{m} \prod_{j=1}^{\eta_{r}}\left(\frac{1}{\beta} z \frac{d}{d z}+\gamma_{r}+j\right)\right] I_{\beta, m}^{\left(\gamma_{k}+\delta_{k}\right),\left(\eta_{k}-\delta_{k}\right)} f(z), \\
\text { and }{ }^{*} D_{\beta, m}^{\left(\gamma_{k}\right),\left(\delta_{k}\right)} f(z)=I_{\beta, m}^{\left(\gamma_{k}+\delta_{k}\right),\left(\eta_{k}-\delta_{k}\right)} D_{\eta} f(z) .
\end{gathered}
$$

Under generalized (multiple, multi-order) fractional derivatives of the R-L type, resp. of the Caputo type, we have in mind all the differ-integral/integro-differential operators of the form

$$
\widetilde{D} f(z)=D_{\left(\beta_{k}\right), m}^{\left(\gamma_{k}\right),\left(\delta_{k}\right)} z^{-\delta_{0}} f(z)=z^{-\delta_{0}} D_{\left(\beta_{k}\right), m}^{\left(\gamma_{k}-\frac{\delta_{0}}{\beta}\right),\left(\delta_{k}\right)} f(z), \quad \widetilde{{ }^{*}} f(z)={ }^{*} D_{\left(\beta_{k}\right), m}^{\left(\gamma_{k}\right),\left(\delta_{k}\right)} z^{-\delta_{0}} f(z) \text { with } \delta_{0} \geq 0 .
$$

A basic formula for the image of a power function in the general case of (18) and (19) (say from Kiryakova [6]) reads as

$$
I_{\left(\beta_{k}\right), m}^{\left(\gamma_{k}\right),\left(\delta_{k}\right)}\left\{z^{p}\right\}=c_{p} z^{p}, \text { with } c_{p}=\prod_{i=1}^{m} \frac{\Gamma\left(\gamma_{i}+1+p / \beta_{i}\right)}{\Gamma\left(\gamma_{i}+\delta_{i}+1+p / \beta_{i}\right)}, \quad \delta_{k} \geq 0, p>-\beta_{k}\left(\gamma_{k}+1\right), k=1, \ldots, m,
$$


and a similar one holds for the generalized fractional derivatives, both analogous to the same formulas for the classical Erdélyi-Kober operators. These results are in the base of the standard techniques applied by other authors for the evaluation of FC operators of special functions, in various particular cases. Using (27), in particular for $p=k=0,1,2, \ldots, n, \ldots$, then interchanging the integration and summation of the power series for a particular special function, the authors of mentioned papers obtain a new power series to be recognized as another special function (in the successful cases, or the result is useless, left just as such series or as some ${ }_{p} \Psi_{q}$-function). Our general result states as follows.

Theorem 1 (Kiryakova, since 1988, see, e.g., ([6,38], Ch.5)). Let the conditions $\beta_{k}\left(\gamma_{k}+1\right)>-\mu, \delta_{k} \geq$ $0, \beta_{k}>0, k=1, \ldots, m$, be satisfied for the parameters of the multiple $E-K$ integral (18). Then, it preserves the class of weighted analytic functions $f(z)$ in a disk $\Delta_{R}$, denoted by $\mathcal{H}_{\mu}\left(\Delta_{R}=\{|z|<R\}\right)$ :

$$
f(z)=z^{\mu} \sum_{k=0}^{\infty} a_{k} z^{k}=z^{\mu}\left(a_{0}+a_{1} z+\ldots\right) \in \mathcal{H}_{\mu}\left(\Delta_{R}\right) \text { with } R=\left\{\limsup _{k \rightarrow \infty} \sqrt[k]{\left|a_{k}\right|}\right\}^{-1} .
$$

Namely, the images of such functions have the same form:

$$
I_{\left(\beta_{k}\right), m}^{\left(\gamma_{k}\right),\left(\delta_{k}\right)} f(z)=z^{\mu} \sum_{k=0}^{\infty} a_{k} b_{k} z^{k} \in \mathcal{H}_{\mu}\left(\Delta_{R}\right), \text { with } b_{k}=\left\{\prod_{i=1}^{m} \frac{\Gamma\left(\gamma_{i}+\frac{k+\mu}{\beta_{i}}+1\right)}{\Gamma\left(\gamma_{i}+\delta_{i}+\frac{k+\mu}{\beta_{i}}+1\right)}\right\}>0,
$$

with the same radius of convergence $R>0$ and the same signs of the coefficients in their series expansions.

\subsection{Some Special Cases of GFC Operators}

We emphasize here only some operators of FC that are recently exploited very often in publications on FC operators of SF. In [6] and the author's other papers as well as in works by other authors, there many other particular cases of linear integral and differential operators provided and used with applications in geometric (univalent) function theory, in differential and integral equations of integer and fractional order, operational calculus, transmutation theory, special functions theory, mathematical models of phenomena of fractional order, etc.

For $m=1$ the kernel-functions of the generalized fractional integrals and derivatives (18) and (23) can be represented as

$$
H_{1,1}^{1,0}\left[\sigma \mid \begin{array}{c}
(\gamma+\delta, 1 / \beta) \\
(\gamma, 1 / \beta)
\end{array}\right]=\beta \sigma^{\beta-1} G_{1,1}^{1,0}\left[\begin{array}{c|c}
\sigma^{\beta} & \gamma+\delta \\
\gamma
\end{array}\right]=\beta \frac{\sigma^{\beta \gamma+\beta-1}\left(1-\sigma^{\beta}\right)^{\delta-1}}{\Gamma(\delta)},
$$

therefore we have the E-K and R-L $(\gamma=0, \beta=1)$ operators of classical FC. Many other integration and differentiation operators introduced and used by different authors appear as special cases of $I_{\beta, 1}^{\gamma, \delta}=I_{\beta}^{\gamma, \delta}$, $D_{\beta, 1}^{\gamma, \delta}=D_{\beta}^{\gamma, \delta}, R^{\delta}$ and $D^{\delta}$.

When $\underline{m}=2$, the kernels $H_{2,2}^{2,0}$ and $G_{2,2}^{2,0}$ reduce to a Gauss hypergeometric lfunction:

$$
\begin{aligned}
& H_{2,2}^{2,0}\left[\sigma \mid \begin{array}{c}
\left(\gamma_{1}+\delta_{1}+1-\frac{1}{\beta}, \frac{1}{\beta}\right),\left(\gamma_{2}+\delta_{2}+1-\frac{1}{\beta}, \frac{1}{\beta}\right) \\
\left(\gamma_{1}+1-\frac{1}{\beta}, \frac{1}{\beta}\right),\left(\gamma_{2}+1-\frac{1}{\beta}, \frac{1}{\beta}\right)
\end{array}\right]=G_{2,2}^{2,0}\left[\begin{array}{c}
\sigma^{\beta} \\
\gamma_{1}+\delta_{1}, \gamma_{2}+\delta_{2} \\
\gamma_{1}, \gamma_{2}
\end{array}\right] \\
& =\frac{\sigma^{\beta \gamma_{2}}\left(1-\sigma^{\beta}\right)^{\delta_{1}+\delta_{2}-1}}{\Gamma\left(\delta_{1}+\delta_{2}\right)}{ }_{2} F_{1}\left(\gamma_{2}+\delta_{2}-\gamma_{1}, \delta_{1} ; \delta_{1}+\delta_{2} ; 1-\sigma^{\beta}\right) .
\end{aligned}
$$

In this case, the generalized fractional integrals are known as hypergeometric fractional integrals, and some of them are introduced and studied by Love, Saxena, Kalla, Saigo (see in next Section 6), Hohlov, etc. 
In the case $\underline{m=3}$, a recently very popular example is with the Marichev-Saigo-Maeda (M-S-M) operators. These FC integration operators are introduced and studied by Marichev [39] and by Saigo et al. [40]. Their kernel-function, the Appel $F_{3}$ function (Horn function)

$$
F_{3}\left(a, a^{\prime}, b, b^{\prime}, c, z, \xi\right)=\sum_{m, n=0}^{\infty} \frac{(a)_{m}\left(a^{\prime}\right)_{n}(b)_{m}\left(b^{\prime}\right)_{n}}{(c)_{m+n}} \frac{z^{m} \xi^{n}}{m ! n !},|z|<1,|\xi|<1 \quad \text { (see, e.g., [7,15]), }
$$

appears as a case of the $G_{3,3}^{3,0}$-function and of $H_{3,3}^{3,0}$-function. Indeed, according to [7], p.727, (2); and as observed in Kiryakova [6], p.21:

$$
\begin{aligned}
& \frac{(1-\sigma)^{c-1}}{\Gamma(c)} F_{3}\left(a, a^{\prime}, b, b^{\prime}, c, 1-\frac{1}{\sigma}, 1-\sigma\right) \\
= & G_{3,3}^{3,0}\left[\begin{array}{c}
\sigma \mid \begin{array}{c}
a+b, c-a^{\prime}, c-b^{\prime} \\
a, b, c-a^{\prime}-b^{\prime}
\end{array}
\end{array}\right]=H_{3,3}^{3,0}\left[\begin{array}{c|c}
(a+b, 1),\left(c-a^{\prime}, 1\right),\left(c-b^{\prime}, 1\right) \\
(a, 1),(b, 1),\left(c-a^{\prime}-b^{\prime}, 1\right)
\end{array}\right], \operatorname{Re} c>0 .
\end{aligned}
$$

Therefore, our generalized fractional integrals reduce in this case to the M-S-M integral operators.

Let $\underline{m \geq 1}$ be an arbitrary integer, but all $\delta^{\prime}$ s are integers, say $\delta_{1}=\ldots=\delta_{m}=1$. Then we have the Bessel type integral and differential operators of arbitrary (higher) integer order, introduced by Dimovski [41] (see also [42]) and named as hyper-Bessel operators by Kiryakova ([6], Ch.3), as shown related to the hyper-Bessel functions of Delerue [43] as their eigenfunctions (see Example 16, in next Section 8). The studies on these operators gave rise to our GFC, since they appeared as "fractional" integrals and derivatives of integer multi-orders $(1,1, \ldots, 1)$ and for $\lambda>0$ their fractional powers have multi-orders $(\lambda, \lambda, \ldots, \lambda)$. In Section 8 , we will discuss also the Gelfond-Leontiev operators generated by the multi-index M-L functions, as more general operators of arbitrary multiplicity $m>1$ and arbitrary fractional multi-order.

As mentioned, here we stress on only a few particular examples of GFC operators $I_{\left(\beta_{k}\right), m}^{\left(\gamma_{k}\right),\left(\delta_{k}\right)}$, $D_{\left(\beta_{k}\right), m}^{\left(\gamma_{k}\right),\left(\delta_{k}\right)}$, that are involved in results on to the topic of this survey. This is because many other authors' works handle the evaluation of images of various elementary or special functions under the classical or some "generalized operators of FC" — such as the operators of R-L, E-K, Saigo, Marichev-Saigo-Maeda. Say, one takes first the cosine or Bessel function, later the generalized Bessel (Bessel-Maitland) function, then an M-L or generalized M-L function, etc., so as to produce new publications by same standard techniques. Very rarely observed, or mostly is ignored, the fact from relation (21) that these are 2-tuple $(m=2)$, respectively 3-tuple $(m=3)$, or $m$-tuple (arbitrary $m>1)$ compositions of Erdélyi-Kober operators. Therefore, the task can be done at once, if one knows how an E-K operator acts on such special functions, all being cases of Wright g.h.f. (4), and then applying the procedure a suitable number of times (2-, 3-, or $m)$. Thus, the result can be predicted in advance, having in mind the general statements in the next sections.

\section{Erdélyi-Kober and Riemann-Liouville Images of $p \Psi_{q}, F_{q}$ and Simpler Special Functions}

Some basic classical results on the topic exist from the previous century that should not be forgotten and on which our approach was built. Namely, the image of a generalized hypergeometric function $F_{q}$, with $p \leq q+1$, under the $R$-L fractional integral/derivative is shown to be the same special function with indices $p$ and $q$ increased by 1 :

$$
R^{\delta}\left\{z^{v-1}{ }_{p} F_{q}\left(a_{1}, \ldots, a_{p} ; b_{1}, \ldots, b_{q} ; \lambda z\right)\right\}=\frac{\Gamma(v)}{\Gamma(\delta+v)} z^{\delta+v-1}{ }_{p+1} F_{q+1}\left(a_{1}, \ldots, a_{p}, v ; b_{1}, \ldots, b_{q}, \delta+v ; \lambda z\right),
$$

with $\operatorname{Re} \delta>0, \operatorname{Re} v>0, p \leq q+1 ; \lambda \neq 0, z \in \mathbb{C}$ and if $p=q+1:|\lambda z|<1$ is additionally required. For this, we can refer to Erdélyi et al. ([44], Vol.2), Ch. XIII, Equations (95)-(97); Askey ([45], p.19), and emphasize the survey by Lavoie-Osler-Tremblay [46], a table on p.261. Then, to make use of (32), in our older works on the topic since 1984-1985, we started from the R-L images of the ${ }_{p} F_{q}$ functions 
with the lowest possible indices: ${ }_{1} F_{0},{ }_{1} F_{1}$ and ${ }_{0} F_{1}$, given by three basic elementary functions, see for example in Kiryakova ([6], Ch.4). Some particular illustrative cases in this direction were mentioned there, as

$$
\begin{gathered}
I_{2}^{-1, v+\frac{1}{2}}\{\cos z\}=R_{z^{2}}^{v+\frac{1}{2}}\left\{\frac{\cos z}{z}\right\}=\sqrt{\pi} 2^{v} z^{-v} J_{v}(z)=\sqrt{\pi} 2^{v-1} z^{-v+1}{ }_{0} F_{1}\left(v+1 ;-\frac{z^{2}}{4}\right), v>-\frac{1}{2}, \\
R^{\delta}\left\{z^{v-1} \exp (\lambda z)\right\}=\frac{\Gamma(v)}{\Gamma(\delta+v)} z^{\delta+v-1}{ }_{1} F_{1}(v ; \delta+v ; \lambda z), \quad \operatorname{Re} \delta>0, \operatorname{Re} v>0, \\
R^{\delta}\left\{z^{v-1}(z-\lambda)^{\mu}\right\}=\frac{(-\lambda)^{\mu} z^{\delta+v-1} \Gamma(v)}{\Gamma(\delta+v)}{ }_{2} F_{1}\left(-\mu, v ; \delta+v, b_{1}, \ldots, b_{q} ; z / \lambda\right), \quad \operatorname{Re} \delta>0, \operatorname{Re} v>0 .
\end{gathered}
$$

Note that (33) is an interpretation of the Poisson integral formula for the Bessel function, that has been generalized in [42] and ([6], Ch.4) to represent the hyper-Bessel functions $J_{v_{1}, \ldots, v_{m}}^{(m)}$ with multi-indices $\left(v_{1}, \ldots, v_{m}\right)$ (the Bessel function $J_{v}$ is the case for $m=q-p=1$ with one index $\left.v\right)$, that is, to represent the ${ }_{0} F_{q-p}$-functions by means of "generalized cosine" $\cos _{m}$.

R-L integrals/ derivatives of the most general G-and $H$-functions are also well known in the literature (for example, from [44]), and these are the same type of functions but with increased orders and additional parameters.

Along with the mentioned old classical results, recently, new articles are published on the evaluation of classical (R-L, E-K) or generalized FC operators of classical SF or of SF of FC almost every day (e.g., in 2020: [47,48]), and also of their multivariate or matrix variants. Just as one example on fractional operators for the matrix Wright hypergeometric functions (5), is a 2020 paper [49].

The classical results (32) and (33)-(35) have been extended in our works (as in ([6], Ch.4), [22,24,50]) in terms of the Erdélyi-Kober operators (12) and (15) and for their counterparts of the GFC: $I_{\left(\beta_{k}\right), m}^{\left(\gamma_{k}\right),\left(\delta_{k}\right)}$, $D_{\left(\beta_{k}\right), m}^{\left(\gamma_{k}\right),\left(\delta_{k}\right)}$, not only for ${ }_{p} F_{q}$ but for ${ }_{p} \Psi_{q}$ as well. To reduce the Wright g.h.f. ${ }_{p} \Psi_{q}$ in the general case to three basic simplest functions with lowest indices $p$ and $q$, we also apply modifications as the Wright-Erdélyi-Kober multiple operators with a Bessel-Maitland kernel-function and in general, GFC operators with $H$-functions like in (20) but with different parameters $1 / \beta_{k}>0$ and $1 / \lambda_{k}>0$ in the upper and low row. Details are in Kiryakova [24].

Now we provide some basic statements necessary for the topic of this survey, repeating in a few lines the ideas of the proofs, so as to clarify the approach used.

Lemma 1. The image of a Wright g.h.f. $\Psi_{q}$ under the Erdélyi-Kober fractional integral (12) is the same type of function in which the indices $p$-and $q$ are increased by one, and so, has two additional parameters:

$$
\left.I_{\beta}^{\gamma, \delta}\left\{z_{p}^{c} \Psi_{q}\left[\begin{array}{c}
\left(a_{1}, A_{1}\right), \ldots,\left(a_{p}, A_{p}\right) \\
\left(b_{1}, B_{1}\right), \ldots,\left(b_{q}, B_{q}\right)
\end{array} \mid \lambda z^{\mu}\right]\right\}=z_{p+1}^{c} \Psi_{q+1}\left[\begin{array}{c}
\left(a_{i}, A_{i}\right)_{1}^{p}(\gamma+1+c / \beta, \mu / \beta) \\
\left(b_{j}, B_{j}\right)_{1}^{q},(\gamma+\delta+1+c / \beta, \mu / \beta)
\end{array}\right] \lambda z^{\mu}\right] .
$$

It is supposed that $\operatorname{Re} \delta>0, \operatorname{Re} \gamma>-1, \mu>0, \lambda \neq 0, c$ is arbitrary (real), and if $p=q+1$, then $\left|\lambda z^{\mu}\right|<1$ is additionally required.

Proof. In a simpler case with $c=0$, this is Lemma 1 from Kiryakova [2]. There, a proof is based on the Formula (44) (Section 4) for the integral (the Mellin transform) of a product of two $H$-functions, since both the ${ }_{p} \Psi_{q}$-function and the kernel of the E-K operator are cases of $H$-functions; compare (5) and Section 2.3. This approach will be discussed later for the more general case of GFC operators.

As a very standard technique, to prove (36), one can use term-by-term integration in series (4), similarly to that in Kilbas ([16], Th.2) for the particular case of R-L integral, with $c:=v-1$ there:

$$
R^{\delta}\left\{z_{p}^{c} \Psi_{q}\left[\begin{array}{c}
\left(a_{1}, A_{1}\right), \ldots,\left(a_{p}, A_{p}\right) \\
\left(b_{1}, B_{1}\right), \ldots,\left(b_{q}, B_{q}\right)
\end{array} \mid \lambda z^{\mu}\right]\right\}=z_{p+1}^{c+\delta} \Psi_{q+1}\left[\begin{array}{c}
\left(a_{i}, A_{i}\right)_{1}^{p},(c+1, \mu) \\
\left(b_{j}, B_{j}\right)_{1}^{q},(c+\delta+1, \mu)
\end{array} \mid \lambda z^{\mu}\right] .
$$


Note that the known simpler formula (32), written by means of E-K integrals, appears as a special case of (36) if all $A_{1}=\ldots=A_{p}=B_{1}=\ldots=B_{q}=1$ and $\beta=1$, see Equation (4.2.2') in Kiryakova [6], for $\operatorname{Re} \delta>0, \operatorname{Re} \gamma>-1$ :

$$
I_{1}^{\gamma, \delta}\left\{{ }_{p} F_{q}\left(a_{1}, \ldots, a_{p} ; b_{1}, \ldots, b_{q} ; \lambda z\right)\right\}=\frac{\Gamma(\gamma+1)}{\Gamma(\gamma+\delta+1)}{ }_{p+1} F_{q+1}\left(a_{1}, \ldots, a_{p}, \gamma+1 ; b_{1}, \ldots, b_{q}, \gamma+\delta+1 ; \lambda z\right) .
$$

Lemma 2. The image of a Wright g.h.f. $\Psi_{q}$ under the E-K fractional derivative (15) is the same kind of function but with indices $p$ and $q$ increased by 1 , and with 2 the additional parameters:

$$
\left.D_{\beta}^{\gamma, \delta}\left\{z_{p}^{c} \Psi_{q}\left[\begin{array}{c}
\left(a_{1}, A_{1}\right), \ldots,\left(a_{p}, A_{p}\right) \\
\left(b_{1}, B_{1}\right), \ldots,\left(b_{q}, B_{q}\right)
\end{array} \mid \lambda z^{\mu}\right]\right\}=z_{p+1}^{c} \Psi_{q+1}\left[\begin{array}{c}
\left(a_{i}, A_{i}\right)_{1}^{p},(\gamma+\delta+1+c / \beta, \mu / \beta) \\
\left(b_{j}, B_{j}\right)_{1}^{q},(\gamma+1+c / \beta, \mu / \beta)
\end{array}\right] \lambda z^{\mu}\right],
$$

provided $\operatorname{Re} \delta>0, \operatorname{Re} \gamma>-1, \mu>0, \lambda \neq 0$, and if $p=q+1$, we require $\left|\lambda z^{\mu}\right|<1$.

Proof. For $c=0$ this is Lemma 3 in Kiryakova [2], and for the case of $\gamma=0, \beta=1$ we have the formula for the R-L fractional derivative from Kilbas ([16], Th.4) (where $v-1:=c, \operatorname{Re} c>-1$, and the same other conditions):

$$
D^{\delta}\left\{z_{p}^{c} \Psi_{q}\left[\begin{array}{c}
\left(a_{1}, A_{1}\right), \ldots,\left(a_{p}, A_{p}\right) \\
\left(b_{1}, B_{1}\right), \ldots,\left(b_{q}, B_{q}\right)
\end{array} \mid \lambda z^{\mu}\right]\right\}=z^{c-\delta}{ }_{p+1} \Psi_{q+1}\left[\begin{array}{c}
\left(a_{i}, A_{i}\right)_{1}^{p},(c+1, \mu) \\
\left(b_{j}, B_{j}\right)_{1}^{q},(c+1-\delta, \mu)
\end{array} \mid \lambda z^{\mu}\right] .
$$

The same standard term-by-term integration/differentiation technique can be used for the proof of (39).

Consider also the simplest case as an analog of (39), when $\beta=1, c=0$ and all $A_{1}=\ldots=A_{p}=$ $B_{1}=\ldots=B_{q}=1$. This is our Lemma 4.3.1 from [6] for the ${ }_{p} F_{q}$-functions. Namely,

$$
D_{1}^{\gamma, \delta}\left\{{ }_{p} F_{q}\left(a_{1}, \ldots, a_{p} ; b_{1}, \ldots, b_{q} ; \lambda z\right)\right\}=\frac{\Gamma(\gamma+\delta+1)}{\Gamma(\gamma+1)}{ }_{p+1} F_{q+1}\left(a_{1}, \ldots, a_{p}, \gamma+\delta+1 ; b_{1}, \ldots, b_{q}, \gamma+1 ; \lambda z\right) .
$$

In the proof of (41) given in [2], we used the relation (16) between the E-K derivative (15) and the R-L derivative $D^{\delta} f(z)=\left(\frac{d}{d z}\right)^{n} R^{n-\delta} f(z), n=[\delta]+1$, combined with the result (32). Then, employed a formula from ([7], Section 7.2.3, (51)) for differentiation of integer order $n$ of a generalized hypergeometric function ${ }_{p} F_{q}$ with specific parameters as above.

Here we demonstrate a new proof of Lemma 2 for the more general case of Wright function ${ }_{p} \Psi_{q}$. For simplicity, $\beta=1$ and $\mu=1$. Interpreting the E-K derivative (15) as in (16), we have subsequently:

$$
\begin{aligned}
& \text { The L.H.S. of }(41)=\left[z^{-\gamma} D^{\delta} z^{\gamma+\delta}\right]\left\{z_{p}^{c} \Psi_{q}\left[\begin{array}{c}
\left(a_{i}, A_{i}\right)_{1}^{p} \\
\left(b_{j}, B_{j}\right)_{1}^{q}
\end{array} \mid \lambda z\right]\right\} \\
& =\left[z^{-\gamma}\left(\frac{d}{d z}\right)^{n} R^{n-\delta}\right]\left\{z^{\gamma+\delta+c_{p} \Psi_{q}}\left[\begin{array}{c}
\left(a_{i}, A_{i}\right)_{1}^{p} \\
\left(b_{j}, B_{j}\right)_{1}^{q}
\end{array} \mid \lambda z\right]\right\}
\end{aligned}
$$

(and due to (37)) $=z^{-\gamma}\left(\frac{d}{d z}\right)^{n}\left\{z_{p+1}^{\gamma+n+c} \Psi_{q+1}\left[\begin{array}{c}\left(a_{i}, A i\right)_{1}^{p},(\gamma+\delta+c+1,1 \\ \left(b_{j}, B_{j}\right)_{1}^{q},(\gamma+n+c+1,1)\end{array} \mid \lambda z\right]\right\}$.

Now, we use the representation (5) of the Wright g.h.f. as an $H$-function, and may apply a formula for differentiation of integer order $n$ of the $H$-function, say Equation (1.69) from Mathai-Saxena-Haubold [51], to continue as follows:

$$
\ldots=z^{-\gamma}\left(\frac{d}{d z}\right)^{n}\left\{\begin{array}{l|c}
z^{\gamma+n+c} H_{p+1, q+2}^{1, p+1}
\end{array}\left[\begin{array}{l|c}
\left(1-a_{i}, A_{i}\right)_{1}^{p},(-\gamma-\delta-c, 1) \\
(0,1),\left(1-b_{j}, B_{j}\right)_{1}^{q},(-\gamma-n-c, 1)
\end{array}\right]\right\}
$$




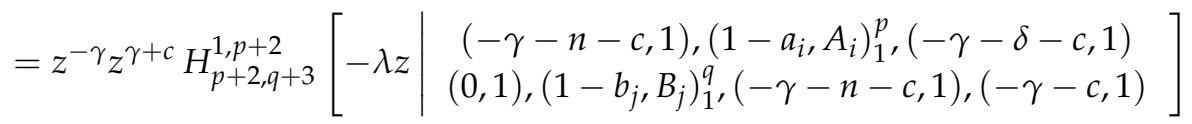

and because of the coincidence of the terms $(-\gamma-n-c, 1)$ in upper and low parameters' rows, according to the reduction order formula for the $H$-function: (1.56) in [51], see also (E.8) in [6], and $[7,9])$, we have

$$
\ldots=z^{c} H_{p+1, q+2}^{1, p+1}\left[\begin{array}{l|l}
-\lambda z & \left(1-a_{i}, A_{i}\right)_{1}^{p},(-\gamma-\delta-c, 1) \\
(0,1),\left(1-b_{j}, B_{j}\right)_{1}^{q},(-\gamma-c, 1)
\end{array}\right],
$$

which, by using again (5) to go back to a Wright g.h.f., gives the result (39). In case $\beta \neq 1$, substitution $z \mapsto z^{1 / \beta}$ is necessary, and same for $\mu \neq 1$.

Yet another approach to check the validity of (39) is to use the identity $D_{\beta}^{\gamma, \delta} I_{\beta}^{\gamma, \delta} f(z)=f(z)$ for $f(z):=z_{p}^{c} \Psi_{q}\left[\begin{array}{c}\left(a_{1}, A_{1}\right), \ldots,\left(a_{p}, A_{p}\right) \\ \left(b_{1}, B_{1}\right), \ldots,\left(b_{q}, B_{q}\right)\end{array} \mid \lambda z^{\mu}\right]$, the result from Lemma 1 and reduction of the intermediate result ${ }_{p+2} \Psi_{q+2}$ to a $p+1 \Psi_{q+1}$-function, since the last two equal parameters in the upper and low rows of its series eliminate each other.

Remark 2. The corresponding result for the Caputo-type E-K derivative ${ }^{*} D_{\beta}^{\gamma, \delta}$ for images of the Wright ${ }_{p} \Psi_{q}$-functions, and in particular also for the ${ }_{p} F_{q}$-functions and for the simpler case of operators with $\beta_{1}=\ldots=$ $\beta_{m}=\beta>0$, will be presented in another separate work.

Example 1. The classical result (34) can be extended to an Erdélyi-Kober integral if we use Lemma 1 for $\exp (z)={ }_{0} F_{0}(-;-; z)={ }_{1} F_{1}(0 ; 0 ; z)={ }_{1} \Psi_{1}\left[\begin{array}{c}(0,1) \\ (0,1)\end{array} \mid z\right]$ (which is also $a_{0} \Psi_{0}, G_{0,1}^{1,0}$ and $H_{0,1}^{1,0}$-function):
$I_{\beta}^{\gamma, \delta}\left\{z^{c} \exp (\lambda z)\right\}=I_{\beta}^{\gamma, \delta}\left\{z^{c}{ }_{1} \Psi_{1}\left[\begin{array}{r}(0,1) \\ (0,1)\end{array} \mid \lambda z\right]\right\}=z^{c}{ }_{2} \Psi_{2}\left[\begin{array}{c}(0,1),(\gamma+1+c / \beta, 1 / \beta) \\ (0,1),(\gamma+\delta+1+c / \beta, 1 / \beta)\end{array} \mid z\right]$
$\quad={ }_{1} \Psi_{1}\left[\begin{array}{c}(\gamma+1+c / \beta, 1 / \beta) \\ (\gamma+\delta+1+c / \beta, 1 / \beta)\end{array} \mid \lambda z\right]$, reducible to (34) for $\gamma=0, \beta=1, v=c+1>0$.

\section{Results for the Generalized Fractional Calculus Operators of Special Functions}

Here we present our results on evaluating operators of generalized fractional calculus (in the sense of [6] and of Riemann-Liouville type) of wide classes of special functions as the Wright generalized hypergeometric functions ${ }_{p} \Psi_{q}$ and even of the Fox $H$-functions (thus incorporating the SF of FC) and in particular, of the ${ }_{p} F_{q}$ - and Meijer G-functions (thus having general results also for the "classical" SF).

We start with the most general result, presented in Kiryakova ([2], Th.3) and mentioned in ([3], Th.4.3).

Theorem 2. The generalized (m-tuple) fractional integral $I_{\left(\beta_{k}\right), m}^{\left(\gamma_{k}\right),\left(\delta_{k}\right)}$ of a H-function is again an H-function:

$$
I_{\left(\beta_{k}\right), m}^{\left(\gamma_{k}\right),\left(\delta_{k}\right)}\left\{H_{u, v}^{s, t}\left[\lambda z \mid \begin{array}{c}
\left(c_{i}, C_{i}\right)_{1}^{u} \\
\left(d_{j}, D_{j}\right)_{1}^{v}
\end{array}\right]\right\}=H_{u+m, v+m}^{s, t+m}\left[\lambda z \mid \begin{array}{c}
\left(c_{i}, C_{i}\right)_{1}^{t},\left(-\gamma_{k}\right)_{1}^{m},\left(c_{i}, C_{i}\right)_{t+1}^{u} \\
\left(d_{j}, D_{j}\right)_{1}^{s},\left(-\gamma_{k}-\delta_{k}\right)_{1}^{m},\left(d_{j}, D_{j}\right)_{s+1}^{v}
\end{array}\right] .
$$

Note that three of the orders of the H-function are increased by the multiplicity $m$, and additional $m+m$ parameters appear depending on those of the operator.

Proof. The following known formula for integral (can be seen as a Mellin transform) of product of two Fox $H$-functions is very important for evaluating integrals of products of special functions of general nature, 
because almost all of them can be presented as $H$-functions (([9], Section 5.1, (5.1.1)), ([7], Section 2.25, (1)), see also (E.21') in ([6], Appendix)):

$$
\begin{aligned}
& \int_{0}^{\infty} \sigma^{\alpha-1} H_{u, v}^{s, t}\left[\varkappa \sigma \mid \begin{array}{c}
\left(c_{i}, C_{i}\right)_{1}^{u} \\
\left(d_{l}, D_{l}\right)_{1}^{v}
\end{array}\right] H_{p, q}^{m, n}\left[\omega \sigma^{r} \mid \begin{array}{c}
\left(a_{j}, A_{j}\right)_{1}^{p} \\
\left(b_{k}, B_{k}\right)_{1}^{q}
\end{array}\right] d \sigma \\
& =\varkappa^{-\alpha} H_{p+v, q+u}^{m+t, n+s}\left[\begin{array}{l|l}
\left(a_{j}, A_{j}\right)_{1}^{n},\left(1-d_{l}-\alpha D_{l}, r D_{l}\right)_{1}^{v},\left(a_{j}, A_{j}\right)_{n+1}^{p} \\
\varkappa^{r} \\
\left(b_{k}, B_{k}\right)_{1}^{m},\left(1-c_{i}-\alpha C_{i}, r C_{i}\right)_{1}^{u},\left(b_{k}, B_{k}\right)_{m+1}^{q}
\end{array}\right],
\end{aligned}
$$

under the conditions $\Delta>-1, a^{*}=\Delta+1>0$ ) (in terms of (2)).

To prove (43) we use the definition (18) of $I_{\left(\beta_{k}\right), m}^{\left(\gamma_{k}\right),\left(\delta_{k}\right)}$, the fact that the kernel $H_{m, m}^{m, 0}$-function vanishes for $|\sigma|>1$ and so the limits $(0,1)$ of the integral can be changed into $(0, \infty)$, and the above Formula $(44)$ :

$$
\begin{aligned}
I_{\left(\beta_{k}\right), m}^{\left(\gamma_{k}\right),\left(\delta_{k}\right)}\left\{H_{u, v}^{s, t}\left[\lambda z \mid \begin{array}{c}
\left(c_{i}, C_{i}\right)_{1}^{u} \\
\left(d_{j}, D_{j}\right)_{1}^{v}
\end{array}\right]\right\}=\int_{0}^{1} \ldots d \sigma=\int_{0}^{\infty} \ldots d \sigma \\
=\int_{0}^{\infty} H_{m, m}^{m, 0}\left[\sigma \mid \begin{array}{c}
\left(\gamma_{k}+\delta_{k}+1-\frac{1}{\beta_{k}}, \frac{1}{\beta_{k}}\right)_{1}^{m} \\
\left(\gamma_{k}+1-\frac{1}{\beta_{k}}, \frac{1}{\beta_{k}}\right)_{1}^{m}
\end{array}\right] H_{u, v}^{s, t}\left[\lambda z \sigma \mid \begin{array}{c}
\left(c_{i}, C_{i}\right)_{1}^{u} \\
\left(d_{j}, D_{j}\right)_{1}^{v}
\end{array}\right] d \sigma \\
=H_{u+m, v+m}^{s+0, t+m}\left[\lambda z \mid \begin{array}{c}
\left(c_{i}, C_{i}\right)_{1}^{t},\left(1-\gamma_{k}-1+\frac{1}{\beta_{k}}-\frac{1}{\beta_{k}}, \frac{1}{\beta_{k}}\right)_{1}^{m},\left(c_{i}, C_{i}\right)_{t+1}^{u} \\
\left(d_{j}, D_{j}\right)_{1}^{s},\left(1-\gamma_{k}-\delta_{k}-1+\frac{1}{\beta_{k}}-\frac{1}{\beta_{k}}, \frac{1}{\beta_{k}}\right)_{1}^{m},\left(d_{j}, D_{j}\right)_{s+1}^{v}
\end{array}\right] \\
\quad=H_{u+m, v+m}^{s, t+m}\left[\begin{array}{c}
\left(c_{i}, C_{i}\right)_{1}^{t},\left(-\gamma_{k}, \frac{1}{\beta_{k}}\right)_{1}^{m},\left(c_{i}, C_{i}\right)_{t+1}^{u} \\
\left(d_{j}, D_{j}\right)_{1}^{s},\left(-\gamma_{k}-\delta_{k}, \frac{1}{\beta_{k}}\right)_{1}^{m},\left(d_{j}, D_{j}\right)_{s+1}^{v}
\end{array}\right] .
\end{aligned}
$$

For $\beta_{1}=\beta_{2}=\ldots=\beta_{m}=\beta>0$, the image of an arbitrary $G$-function under the simpler GFC integrals with Meijer's $G_{m, m}^{m, 0}$-kernels has been provided earlier.

Corollary 1 (Lemma 1.2.2, [6])). The $I_{\beta, m}^{\left(\gamma_{k}\right),\left(\delta_{k}\right)}$-image of a G-function is also a G-function in which the three orders are increased by the multiplicity $m$ and has additional $m+m$ parameters depending on those of the GFC operator:

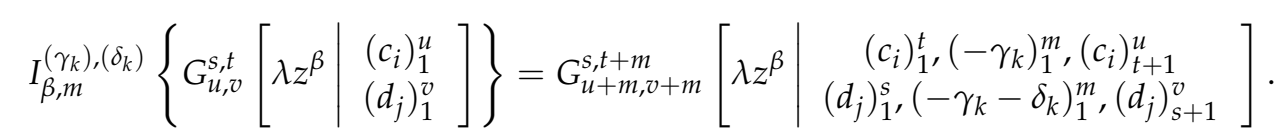

Proof. In this case one can use a formula for the integral of product of two arbitrary G-functions, simpler than (44) (see for example, ([7], Section 2.24, (1)]) and with a proof in ([6], App., (A.29))), and be reminded again that the $G_{m, m}^{m, 0}$-function vanishes outside the unit disc. Thus the integral (45)

$$
I=\int_{0}^{1} \ldots d \sigma=\int_{0}^{\infty} G_{m, m}^{m, 0}\left[\sigma \mid \begin{array}{c}
\left(\gamma_{k}+\delta_{k}\right)_{1}^{m} \\
\left(\gamma_{k}\right)_{1}^{m}
\end{array}\right] G_{u, v}^{s, t}\left[\lambda z^{\beta} \mid \begin{array}{c}
\left(c_{i}\right)_{1}^{u} \\
\left(d_{j}\right)_{1}^{v}
\end{array}\right] d \sigma,
$$

gives the required image $G$-function. Because the $G_{u, v}^{s, t}$-function is from the space of an analytic function in a disk centered at the origin, and has the following asymptotic behavior

$$
G_{u, v}^{s, t}\left[\lambda z^{\beta}\right]=O\left(z^{d^{*}}\right) \text { as }|z| \rightarrow 0, \text { with } d^{*}=\beta \min _{j} d_{j}>\max _{k}\left[-\beta\left(\gamma_{k}+1\right)\right],
$$

the conditions for the used formula to hold on are satisfied. 
Formulas (43) and (45) can be used to evaluate practically all (classical and generalized) operators of FC of arbitrary SF (which are representable either as a $G$ - or as a more general $H$-function).

Now we present the main result on the topic of this survey paper, which comes from Kiryakova [2], Th.1.

Theorem 3. Assume that the conditions $\delta_{k} \geq 0, \gamma_{k}>-1, \beta_{k}>0, k=1, \ldots, m$ and $\mu>0, \lambda \neq 0$ hold. The image of a Wright g.h.f. ${ }_{p} \Psi_{q}(z)$ by a generalized fractional integral (20) (multiple, m-tuple Erdélyi-Kober integral) is another Wright g.h.f. with indices $p$ and $q$ increased by the multiplicity $m$ and with additional parameters coming from those of the GFC integral:

$$
\begin{aligned}
& I_{\left(\beta_{k}\right)_{1}^{m}, m}^{\left(\gamma_{k}\right)^{m},\left(\delta_{k}\right)_{1}^{m}}\left\{z_{p}^{c} \Psi_{q}\left[\begin{array}{c}
\left(a_{1}, A_{1}\right), \ldots,\left(a_{p}, A_{p}\right) \\
\left(b_{1}, B_{1}\right), \ldots,\left(b_{q}, B_{q}\right)
\end{array} \mid \lambda z^{\mu}\right]\right\} \\
= & z_{p+m}^{c} \Psi_{q+m}\left[\begin{array}{c}
\left(a_{i}, A_{i}\right)_{1}^{p},\left(\gamma_{i}+1+\frac{c}{\beta_{i}}, \frac{\mu}{\beta_{i}}\right)_{1}^{m} \\
\left(b_{j}, B_{j}\right)_{1}^{q},\left(\gamma_{i}+\delta_{i}+1+\frac{c}{\beta_{i}}, \frac{\mu}{\beta_{i}}\right)_{1}^{m}
\end{array} \mid \lambda z^{\mu}\right] .
\end{aligned}
$$

Proof. Here we briefly repeat the proof from Kiryakova [2], Th.1, in order to exhibit the main ideas on which this survey paper is based.

As one approach to prove (46), the general integral formula (44) can be used. This theorem can be seen also as a consequence of Theorem 2. It is because the kernel-function of the operator is a $H_{m, m}^{m, 0}$-function and the ${ }_{p} \Psi_{q}$-function is a $H_{p, q+1}^{1, p}$-function, see (5). Then, according to (45) the result will be a $H_{p+m, q+1+m}^{1, p+m}$-function that should be recognized as a $p+1 \Psi_{q+1}$-function, because it is reduced to a $H_{p+1, q+2}^{1, p+1}$-function in view of the coincidence of $(m-1)$ parameters in the upper and low row (use "reduction order" property of the $H$-function, [7], Section 8.3, 6.; [6], App. (E.8), etc.).

However, to clarify our main idea it is more instructive to refer to the decomposition property (21) presenting the generalized fractional integral (18) as a product of commuting (classical) Erdélyi-Kober operators. In the simplest case, we use subsequently $m$-times (36) from Lemma 1, to get:

$$
\begin{aligned}
& I_{\left(\beta_{k}\right)_{1}^{m}, m}^{\left.\left(\gamma_{k}\right)^{m}, \delta_{k}\right)_{1}^{m}}\left\{{ }_{p} \Psi_{q}\left[\begin{array}{c}
\left(a_{i}, A_{i}\right)_{1}^{p} \\
\left(b_{j}, B_{j}\right)_{1}^{q}
\end{array} \mid \lambda z\right]\right\}=I_{\left(\beta_{k}\right)_{1}^{m-1}, m-1}^{\left(\gamma_{k} m-1,\left(\delta_{k}\right)_{1}^{m-1}\right.}\left\{I_{\beta_{m}, \gamma_{p}, \delta_{m} \Psi_{q}}^{\gamma_{1}}\left[\begin{array}{c}
\left(a_{i}, A_{i}\right)_{1}^{p} \\
\left(b_{j}, B_{j}\right)_{1}^{q}
\end{array} \mid \lambda z\right]\right\} \\
& =I_{\left(\beta_{k}\right)_{1}^{m-2}, m-2}^{\left(\gamma_{k}\right)^{m-2},\left(\delta_{k}\right)_{1}^{m-2}}\left\{I_{\beta_{m-1}}^{\gamma_{m-1}, \delta_{m-1}}\left\{p+1 \Psi_{q+1}\left[\begin{array}{c}
\left(a_{i}, A_{i}\right)_{1}^{p},\left(\gamma_{m}+1,1 / \beta_{m}\right) \\
\left(b_{j}, B_{j}\right)_{1}^{q},\left(\gamma_{m}+\delta_{m}+1,1 / \beta_{m}\right)
\end{array} \mid \lambda z\right]\right\}\right\} \\
& =\cdots=I_{\beta_{1}}^{\gamma_{1}, \delta_{1}}\left\{I_{\beta_{2}, \delta_{2}}^{\gamma_{2}}\left\{p+m-2 \Psi_{q+m-2}\left[\begin{array}{c}
\left(a_{i}, A_{i}\right)_{1}^{p},\left(\gamma_{r}+1,1 / \beta_{r}\right)_{1}^{m-2} \\
\left(b_{j}, B_{j}\right)_{1}^{q},\left(\gamma_{r}+\delta_{r}+1,1 / \beta_{r}\right)_{1}^{m-2}
\end{array} \mid \lambda z\right]\right\}\right\} \\
& =I_{\beta_{1}}^{\gamma_{1}, \delta_{1}}\left\{p_{p+m-1} \Psi_{q+m-1}\left[\begin{array}{c}
\left(a_{i}, A_{i}\right)_{1}^{p},\left(\gamma_{r}+1,1 / \beta_{r}\right)_{1}^{m-1} \\
\left(b_{j}, B_{j}\right)_{1}^{q},\left(\gamma_{r}+\delta_{r}+1,1 / \beta_{r}\right)_{1}^{m-1}
\end{array} \mid \lambda z\right]\right\} \\
& ={ }_{p+m} \Psi_{q+m}\left[\begin{array}{c|c}
\left(a_{i}, A_{i}\right)_{1}^{p},\left(\gamma_{r}+1,1 / \beta_{r}\right)_{1}^{m} & \lambda z \\
\left(b_{j}, B_{j}\right)_{1}^{q},\left(\gamma_{r}+\delta_{r}+1,1 / \beta_{r}\right)_{1}^{m} &
\end{array}\right] .
\end{aligned}
$$

To derive the general relation (46) we apply to the above result the property for "generalized commutation" from Kiryakova ([6], Ch.5, (5.1.28)), namely:

$$
I_{\left(\beta_{k}\right), m}^{\left(\gamma_{k}\right),\left(\delta_{k}\right)} z^{c} f\left(z^{\mu}\right)=z^{c} I_{\left(\frac{\beta_{k}}{m}\right), m}^{\left(\gamma_{k}+\frac{c}{\beta_{k}}\right),\left(\delta_{k}\right)} f\left(z^{\mu}\right), \text { with } \mu>0 .
$$


Corollary 2. (Lemma 4.2.1., Equations (4.2.2)-(4.2.2') in Kiryakova ([6], Ch.4)) The image of a $p F_{q}$ g.h.f. (6) under a generalized (m-tuple) fractional integral $I_{1, m}^{\left(\gamma_{k}, \delta_{k}\right)}:=I_{(1, \ldots, 1), m^{\prime}}^{\left(\gamma_{k}, \delta_{k}\right)}$ (19) with (for simplicity) all $\beta_{k}=\beta=$ $1, k=1, \ldots, m$, is another g.h.f. of the same kind with indices increased by the multiplicity $m$ :

$$
I_{1, m}^{\left(\gamma_{k}\right)_{1}^{m},\left(\delta_{k}\right)_{1}^{m}}\left\{p F_{q}\left(a_{1}, \ldots, a_{p} ; b_{1}, \ldots, b_{q} ; \lambda z\right)\right\}={ }_{p+m} F_{q+m}\left(a_{1}, \ldots, a_{p},\left(\gamma_{i}+1\right)_{1}^{m} ;\left(b_{1}, \ldots, b_{q},\left(\gamma_{i}+\delta_{i}+1\right)_{1}^{m} ; \lambda z\right) .\right.
$$

The above results (46) and (49) can be interpreted alternatively as the assertions stated in our earlier works ([6,50], Ch.4) (in the simpler case of Corollary 2), and later in [24] (in more general case of Theorem 3). That is, a $p+m \Psi_{q+m}$-function (resp. a $p+m F_{q+m}$-function) of the form below can be represented by means of a multiple ( $m$-tuple) operator of GFC

$$
\widetilde{I}=I_{\left(1 / \beta_{i}\right)_{i=1}^{m}, m}^{\left(a_{p+i}-1\right)_{i=1}^{m},\left(b_{q+i}-a_{p+i}\right)_{i=1}^{m}}
$$

of a $p \Psi_{q}$-function (resp. a $p F_{q}$-function), with orders reduced by $m$, namely:

$$
\left.p+m \Psi_{q+m}\left[\begin{array}{cc}
\left(a_{i}, A_{i}\right)_{i=1}^{p} ; & \left(a_{p+i}, 1 / \beta_{i}\right)_{i=1}^{m} \\
\left(b_{j}, B_{j}\right)_{k=1}^{q} ; & \left(b_{q+i}, 1 / \beta_{i}\right)_{i=1}^{m}
\end{array} \mid \lambda z\right]=\widetilde{I}\left\{\Psi_{q}\left[\begin{array}{c}
\left(a_{j}, A_{j}\right)_{j=1}^{p} \\
\left(b_{k}, B_{k}\right)_{k=1}^{q}
\end{array}\right] \lambda z\right]\right\} .
$$

In the case of Wright function with arbitrary parameters $A_{p+i}, B_{q+i}, i=1, \ldots, m$ : $p+m \Psi_{q+m}\left[\begin{array}{cc}\left(a_{i}, A_{i}\right)_{i=1}^{p} ; & \left(a_{p+i}, A_{p+i}\right)_{i=1}^{m} \\ \left(b_{j}, B_{j}\right)_{k=1}^{q} ; & \left(b_{q+i}, B_{q+i}\right)_{i=1}^{m}\end{array} \mid z\right]$, such kind of result is presented in [24] by means of more general operators $\widetilde{I}$, the so-called Wright-Erdélyi-Kober operators. This means that using a suitable number of times of a procedure similar to that in proof of Theorem 3, from any $p \Psi_{q}$-function (resp. ${ }_{p} F_{q}$-function) we can go down to one of the three basic generalized hypergeometric functions, depending on if $p<q, p=q$ or $p=q+1:{ }_{0} \Psi_{q-p,{ }_{1}} \Psi_{1},{ }_{2} \Psi_{1}$; resp. to: ${ }_{0} F_{q-p}$ (hyper-Bessel f. and $\cos _{m}-\mathrm{f}$.), ${ }_{1} F_{1}$ (confluent h.f. and exp-f.), ${ }_{2} F_{1}$ (Gauss f. and beta-distribution of form $z^{\alpha}(1-z)^{\beta}$ ). This is the reason that we classified the g.h.f. to be of three basic types, as: "g.h.f. of Bessel/cosine type", "g.h.f. of confluent/exp type" and "g.h.f. of Gauss/beta-distribution type". Details on this approach and such a classification of the SF can be found in Kiryakova ([6,22,24,50], Ch.4).

Analogously to Theorem 3, we have also a relation (image) for the generalized fractional derivatives of g.h.f., presented as Theorem 2 in Kiryakova [2]. The more general formula (as below) is available in Kiryakova, ([3], Theorem 4.2.).

Theorem 4.

$$
\begin{aligned}
& D_{\left(\beta_{k}\right)_{1}^{m}, m}^{\left(\gamma_{k}\right)^{m},\left(\delta_{k}\right)_{1}^{m}}\left\{z_{p}^{c} \Psi_{q}\left[\begin{array}{c}
\left(a_{1}, A_{1}\right), \ldots,\left(a_{p}, A_{p}\right) \\
\left(b_{1}, B_{1}\right), \ldots,\left(b_{q}, B_{q}\right)
\end{array} \mid \lambda z^{\mu}\right]\right\} \\
= & z_{p+m}^{c} \Psi_{q+m}\left[\begin{array}{c}
\left(a_{i}, A_{i}\right)_{1}^{p},\left(\gamma_{k}+\delta_{k}+1+\frac{c}{\beta_{k}}, \frac{\mu}{\beta_{k}}\right)_{1}^{m} \\
\left(b_{j}, B_{j}\right)_{1}^{q},\left(\gamma_{k}+1+\frac{c}{\beta_{k}}, \frac{\mu}{\beta_{k}}\right)_{1}^{m}
\end{array} \mid \lambda z^{\mu}\right] .
\end{aligned}
$$

Proof. One possible approach to derive this, is to use a decomposition formula for the generalized (multiple) fractional derivatives (23),

$$
D_{\left(\beta_{k}\right), m}^{\left(\gamma_{k}\right),\left(\delta_{k}\right)} f(z)=D_{\beta_{m}}^{\gamma_{m}, \delta_{m}}\left\{D_{\beta_{m-1}}^{\gamma_{m-1}, \delta_{m-1}}\left[\cdots D_{\beta_{1}}^{\gamma_{1}, \delta_{1}} f(z)\right]\right\}
$$

as sequential derivatives. Then, we apply $m$-times the result (39) of Lemma 2.

We can verify (51) also directly, in the same way as in the end of the proof of Lemma 2, using the basic relation $D_{\left(\beta_{k}\right), m}^{\left(\gamma_{k}\right),(\delta)_{k}} I_{(\beta)_{k}, m}^{\left(\gamma_{k}\right),\left(\delta_{k}\right)} f(z)=f(z)$.

The case of images under the Caputo type generalized fractional derivatives $D_{\left(\beta_{k}\right)_{1}^{m}, m}^{\left(\gamma_{k}\right)^{m},\left(\delta_{k}\right)_{1}^{m}}$ defined in (24) will be discussed in a separate work, see also Remark 2. 


\section{Examples of Erdélyi-Kober and Riemann-Liuoville Operators of Some Special Functions}

In the beginning of Section 3 we already acknowledged the contributions by some classical authors, such as Erdélyi et al., Askey, Lavoie-Osler-Tremblay, to provide the images of some special and elementary functions under the Riemann-Liuoville fractional integral/derivative, see Formulas (32)-(35). We may refer also to works where detailed tables of images under Riemann-Liuoville operators are provided, for example the book Erdélyi et al. [44], some recent surveys, including in this Journal, such as by Rogosin [18] (as for M-L type functions), Garrappa-Kaslik-Popolizio [52] (images of elementary functions expressed by M-L functions).

As mentioned in Section 3, the proof of Lemma 1, a most general result for Riemann-Liuoville operators of special functions (in sense of g.h.f.) is formula (37) from Kilbas ([16], Th.2):

$$
R^{\delta}\left\{z^{c}{ }_{p} \Psi_{q}\left[\begin{array}{c}
\left(a_{1}, A_{1}\right), \ldots,\left(a_{p}, A_{p}\right) \\
\left(b_{1}, B_{1}\right), \ldots,\left(b_{q}, B_{q}\right)
\end{array} \mid \lambda z^{\mu}\right]\right\}=z^{c+\delta}{ }_{p+1} \Psi_{q+1}\left[\begin{array}{c}
\left(a_{i}, A_{i}\right)_{1}^{p},(c+1, \mu) \\
\left(b_{j}, B_{j}\right)_{1}^{q},(c+\delta+1, \mu)
\end{array} \mid \lambda z^{\mu}\right],
$$

and for the R-L derivative, the corresponding result is as in Equation (40).

It may be instructive to repeat (as from [2]) some very special cases of the images (37) and (40) under the R-L integral $R^{\delta} f(z)=z^{\delta} I_{1}^{0, \delta} f(z)$, that have been derived by the cited authors by the standard term-by-term integration/differentiation. Naturally, these come also as specifications of our results from Lemmas 1 and 2 for the Erdélyi-Kober operators (case $m=1$ ).

Example 2. The R-L fractional integral of the weighted Bessel function, for $\operatorname{Re} \delta>0, \operatorname{Re} v>-1, \operatorname{Re}(\gamma+v)>0$, is given by Kilbas-Sebastian ([53], Cor.1, (28)), in the form

$$
R^{\delta}\left\{z^{\gamma-1} J_{v}(z)\right\}=\frac{z^{\gamma+v+\delta-1}}{2^{v}}{ }_{1} \Psi_{2}\left[\begin{array}{c|c}
(\gamma+v, 2) \\
(\gamma+v+\delta, 2),(v+1,1)
\end{array} \mid-\frac{1}{4} z^{2}\right] .
$$

We can use $J_{v}(z)={ }_{0} \Psi_{1}\left[\begin{array}{c}-- \\ (v+1,1)\end{array} \mid-\frac{1}{4} z^{2}\right]$, so to see (52) as an immediate corollary of (37), and also of our $E-K$ result (36). Because a 1-tuple fractional calculus operator ( $R-L$, or $E-K)$ is applied, the preliminary expectation is confirmed to have as a result $a_{0+1} \Psi_{1+1}$-function.

Example 3. The R-L fractional integral of the generalized Bessel function $J_{v}^{\kappa}$ (usually called Bessel-Maitland function, a name that should correctly be called the Bessel-Wright function) is derived in Kilbas ([16], Th.8, (26)), and extends the above formula (52): Note the representation $J_{v}^{\kappa}(z)={ }_{0} \Psi_{1}\left[\begin{array}{c}-- \\ (v+1, \kappa)\end{array} \mid-z\right]$, then from our result in Lemma 1, and in particular, from (37), it is expected to have the result as $a_{1} \Psi_{2}$-function:

$$
R^{\delta}\left\{z^{\gamma-1} J_{v}^{\kappa}\left(\lambda z^{\mu}\right)\right\}=z^{\gamma+\delta-1}{ }_{1} \Psi_{2}\left[\begin{array}{c}
(\gamma, \mu) \\
(\gamma+\delta, \mu),(v+1, \kappa)
\end{array} \mid-\lambda z^{\mu}\right],
$$

for $\operatorname{Re} \delta>0, \operatorname{Re}(\gamma-1)>-1, \kappa>-1, \mu>0$. The sign "minus" in the argument of RHS was missing in [16] due to a possible typo.

The same result, in terms of the (classical) Wright function is presented in the same paper, Kilbas ([16], Th.6, (18)), with the true argument sign (we slightly change the denotations to be similar as in the first row of our (11)),

$$
R^{\delta}\left\{z^{\gamma-1} \phi\left(\kappa, v+1 ; \lambda z^{\mu}\right)\right\}=z_{1}^{\gamma+\delta-1} \Psi_{2}\left[\begin{array}{c|c}
(\gamma, \mu) \\
(\gamma+\delta, \mu),(v+1, \kappa)
\end{array} \mid \lambda z^{\mu}\right] .
$$


A more useful result, in the sense that the $R$-L integral transforms a generalized Bessel function/resp. Wright function, into same kind of function but with increased index comes if we put $\gamma-1=\nu, \kappa=\mu$ in (53), see, for example, ([16], Cor.8.1, (28)), wth $\operatorname{Re} \delta>0, \operatorname{Rev}>-1, \mu>0$ :

$$
R^{\delta}\left\{z^{v} J_{v}^{\mu}\left(\lambda z^{\mu}\right)\right\}=z^{v+\delta} J_{v+\delta+1}^{\mu}\left(\lambda z^{\mu}\right), \quad R^{\delta}\left\{z^{v} \phi\left(\mu, v+1 ; \lambda z^{\mu}\right)\right\}=z^{v+\delta} \phi\left(\mu, v+\delta+1 ; \lambda z^{\mu}\right), \quad \lambda \neq 0 .
$$

Next, we mention an example with the so-called generalized M-series. Namely, (M.) Sharma and Jain [54] introduced the special function $p M_{q}^{\alpha, \beta}(z)$, as an extension of both g.h.f. $p F_{q}(z)$ and the (2-parameters) M-L function $E_{\alpha, \beta}(z)$ :

$$
\left.\underset{p}{\alpha, \beta} M_{q}\left(a_{1}, \ldots, a_{p} ; b_{1}, \ldots s, b_{q} ; z\right)=\sum_{k=0}^{\infty} \frac{\left(a_{1}\right)_{k} \ldots\left(a_{p}\right)_{k}}{\left(b_{1}\right)_{k} \ldots\left(b_{q}\right)_{k}} \frac{z^{k}}{\Gamma(\alpha k+\beta)}=\kappa_{p+1} \Psi_{q+1}\left[\begin{array}{c}
\left(a_{1}, 1\right), \ldots,\left(a_{p}, 1\right),(1,1) \\
\left(b_{1}, 1\right), \ldots,\left(b_{q}, 1\right),(\beta, \alpha)
\end{array}\right] z\right] .
$$

Here $z, \alpha, \beta \in \mathbb{C}, \operatorname{Re} \alpha>0, p \leq q$ are the integer orders, and if $p=q+1$ we require additionally that $|z|<R=\alpha^{\alpha}$, and $\kappa:=\prod_{j=1}^{q} \Gamma\left(b_{j}\right) / \prod_{i=1}^{p} \Gamma\left(a_{i}\right)$. Usually the following particular cases are always mentioned: (1) $\beta=1$ : this is the (simpler) $M$-series, introduced by M. Sharma (2008, in same journal as [54]); (2) $p=q=0$ (that is, no upper and no lower parameters): this is the M-L function $E_{\alpha, \beta}(z)$; (3) $p=0, q=1, b_{1}=1$ : one has the Wright function $\phi(\alpha, \beta, z)$, or the generalized Bessel-Maitland function; (4) $p=q=1, a_{1}=\gamma, b_{1}=1$ : this is the Prabhakar M-L type function (8), (5) $\alpha=\beta=1$ : we have the g.h.f. $F_{q}\left(a_{1}, \ldots, a_{p} ; b_{1}, \ldots, b_{q} ; z\right)$, etc.

Since (56) is a ${ }_{p+1} \Psi_{q+1}$-function, all FC operators of the form (20) (and their particular cases as R-L, E-K, Saigo, M-S-M) of the M-series can be evaluated using our formulas in Lemmas 1 and 2 and Theorems 3 and 4.

Example 4. In [54], the images of the generalized $M$-series are derived in the case of $R$-L fractional integral and derivative of order $\delta>0$ :

$$
\begin{aligned}
& R^{\delta}\left\{{ }_{p}^{\alpha, \beta} M_{q}\left(a_{1}, \ldots, a_{p} ; b_{1}, \ldots, b_{q} ; z\right)\right\}=\frac{z^{\delta}}{\Gamma(1+\delta)} p+1 \stackrel{\alpha, \beta}{M}_{q+1}\left(a_{1}, \ldots, a_{p}, 1 ; b_{1}, \ldots, b_{q}, 1+\delta ; z\right), \\
& D^{\delta}\left\{{ }_{p}^{\alpha, \beta} M_{q}\left(a_{1}, \ldots, a_{p} ; b_{1}, \ldots, b_{q} ; z\right)\right\}=\frac{z^{-\delta}}{\Gamma(1-\delta)} p+1 \stackrel{\alpha, \beta}{M}_{q+1}\left(a_{1}, \ldots, a_{p}, 1 ; b_{1}, \ldots, b_{q}, 1-\delta ; z\right),
\end{aligned}
$$

using term-by-term integration/differentiation of the series (56). However, having in mind the representations in both sides as Wright g.h.f., one can get these results directly from the corollaries of Lemmas 1 and 2, the $R-L$ integral (37) and derivative (40). See also in Lavault [55].

The formulas in Theorems 3 and 4 can easily be reduced to corresponding results for generalized fractional integrals and derivatives (20) and (23) of the $M$-series, to appear in terms of $p+m M_{q+m}(z)$, with additional parameters depending on $\left(\gamma_{k}\right)_{1}^{m},\left(\delta_{k}\right)_{1}^{m}$. Again in view of (56), evaluation of other particular FC operators, such as E-K, Saigo, M-S-M, of the M-series can be done. For example, the M-S-M images were evaluated by Kumar and Saxena [56].

\section{Saigo Hypergeometric Operators of Various Special Functions}

In a series of papers since 1978, such as [57] (for more references see in [6,58]), Saigo introduced a linear integral operator with Gauss function in the kernel, and applied it first for studying BVP for PDE as the Euler-Darboux equation. Later on, this operator was used by him and collaborators in geometric function theory (classes of univalent functions). It happens that, as a case of the hypergeometric integral operators, the Saigo operator has also a role as an FC operator and this has recently become a reason for great interest for researchers in FC, and mainly to authors whose job is to evaluate images of Saigo operator(s) of various special functions. A search in Google for the phrase "Saigo operator" 
+ "function" returns now more than 1060 results (of course some of them may also concern the more general Marichev-Saigo-Maeda, discussed in next Section 7).

First, let us remind the definition and two basic properties of the Saigo operators.

For complex $\alpha, \beta, \eta$ and $\operatorname{Re} \alpha>0$, the Saigo fractional integration operator (the LHS version) is

$$
\begin{aligned}
& I^{\alpha, \beta, \eta} f(z)=\frac{z^{-\alpha-\beta}}{\Gamma(\alpha)} \int_{0}^{z}(z-\xi)^{\alpha-1}{ }_{2} F_{1}\left(\alpha+\beta,-\eta ; \alpha ; 1-\frac{\xi}{z}\right) f(\xi) d \xi \\
= & \frac{z^{-\beta}}{\Gamma(\alpha)} \int_{0}^{1} s(1-\sigma)^{\alpha-1}{ }_{2} F_{1}(\alpha+\beta,-\eta ; \alpha ; 1-\sigma) f(z \sigma) d \sigma,
\end{aligned}
$$

and we skip the discussion on the RHS versions of the Saigo integrals, as similar. The Saigo fractional derivative is used as: $D^{\alpha, \beta, \eta} f(z)=(d / d z)^{n} I^{\alpha+n, \beta-n, \eta-n} f(z)$ with $n=[-\operatorname{Re} \alpha]+1$. For its explicit differ-integral expression, see for example in ([6], Ch.1). More details can be found in Kiryakova ([6,58], Ch.1, Ch.5) and other our papers dealing with these operators in classes of univalent functions (some of which are joined with Professor Megumi Saigo). A basic formula (known from the original Saigo works) that all authors use (and sometimes derive again) is for the image of a power function:

$$
I^{\alpha, \beta, \eta}\left\{z^{p}\right\}=[\Gamma(p+1) \Gamma(p+\eta-\beta+1) / \Gamma(p-\beta+1) \Gamma(p+\alpha+\eta+1)] z^{p-\beta},
$$

for $\operatorname{Re} \alpha>0, \operatorname{Re}(p+1)>\max [0, \operatorname{Re}(\beta-\eta)]$.

As mentioned in Section 2.3, the Saigo operators are cases of the hypergeometric operators of FC, and of the GFC operators for $m=2$, simply because according to (30) the Gauss kernel function is representable as the kernel of (19) and (18) with $m=2, \beta=1, \gamma_{1}=\eta-\beta, \gamma_{2}=0, \delta_{1}=-\eta, \delta_{2}=\alpha+\eta$ :

$$
\frac{(1-\sigma)^{\alpha-1}}{\Gamma(\alpha)}{ }_{2} F_{1}(\alpha+\beta,-\eta ; \alpha ; 1-\sigma)=G_{2,2}^{2,0}\left[\begin{array}{c}
\sigma \\
-\beta, \alpha+\eta \\
\eta-\beta, 0
\end{array}\right] .
$$

Thus, the Saigo operator is a generalized (2-tuple fractional integral) of the form (20) and therefore in view of (21), it is also a commutable composition of two classical E-K fractional integrals, see for example ([6], Ch.1):

$$
\begin{gathered}
I^{\alpha, \beta, \eta} f(z)=z^{-\beta} I_{(1,1), 2}^{(\eta-\beta, 0),(-\eta, \alpha+\eta)} f(z)=z^{-\beta} I_{1}^{\eta-\beta,-\eta} I_{1}^{0, \alpha+\eta} f(z) \\
=I_{1}^{\eta,-\eta} I_{1}^{\beta, \alpha+\eta} z^{-\beta} f(z)=I_{(1,1), 2}^{(\eta, \beta),(-\eta, \alpha+\eta)} z^{-\beta} f(z)=R^{-\eta} z^{-\alpha-\beta} R^{\alpha+\eta} f(z) .
\end{gathered}
$$

The relation between the first and second lines follows by application of the "generalized commutation" between (multiple) Erdélyi-Kober operators and power functions (([6], Ch.1, (1.3.3)), ([34], Th.4), etc.). For particular parameters $\alpha, \beta, \eta$, the Saigo operator can reduce to one E-K operator or an R-L operator, say for $\beta=-\alpha, \eta=0$ it is an R-L integral; and for $\eta=-\alpha$, an E-K integral: $I^{\alpha, \beta,-\alpha}=z^{-\beta} I_{1}^{-\alpha-\beta, \alpha}$.

Therefore, the Saigo image of some special function, which can be represented as a Wright function ${ }_{p} \Psi_{q}$, can be written as a particular case of the general formulas (46), resp. (51), or also, as a subsequent two-times application of classical E-K operators. Therefore, the Saigo image of $a_{p} \Psi_{q}$-function can always be predicted to result into $a_{p+2} \Psi_{q+2}$-function (unless some parameters in upper and lower rows eliminate each other, and so the indices can be reduced). Our result, as a corollary of Theorem 3 and Corollary 2 states as follows.

Lemma 3. The images of the Wright g.h.f. $p \Psi_{q}$, and in particular of the g.h.f. $p F_{q}$, under the Saigo operator (59) are the same kind of functions with orders increased by 2:

$$
I^{\alpha, \beta, \eta}\left\{z^{c} p_{q}\left[\begin{array}{c}
\left(a_{i}, A_{i}\right)_{1}^{p} \\
\left(b_{j}, B_{j}\right)_{1}^{q}
\end{array} \mid \lambda z^{\mu}\right]\right\}=z^{c-\beta}{ }_{p+2} \Psi_{q+2}\left[\begin{array}{c}
\left(a_{i}, A_{i}\right)_{1}^{p},(\eta-\beta+1+c, \mu),(1+c, \mu) \\
\left(b_{j}, B_{j}\right)_{1}^{q},(-\beta+1+c, \mu),(\alpha+\eta+1+c, \mu)
\end{array} \mid \lambda z^{\mu}\right],
$$


(for $c=0, \mu=1$, this is Cor. 3 in [2]) and

$$
I^{\alpha, \beta, \eta}\left\{{ }_{p} F_{q}\left(a_{1}, \ldots, a_{p} ; b_{1}, \ldots, b_{q} ; \lambda z\right)\right\}=z_{p+2}^{-\beta} F_{q+2}\left(a_{1}, \ldots, a_{p}, \eta-\beta+1,1 ; b_{1}, \ldots, b_{q},-\beta+1, \alpha+\eta+1 ; \lambda z\right),
$$

under the mentioned conditions in the definition of (59).

The following examples for Saigo operators of particular functions from our previous papers [2,3] are repeated here as an illustration for the general result in Lemma 3.

Example 5. The Saigo fractional integral (59) of a weighted Bessel function was evaluated in Kilbas-Sebastian ([53], Th.1), for $\operatorname{Re} \alpha>0, \operatorname{Re} v>-1, \operatorname{Re}(\gamma+v)>\max [0, \operatorname{Re}(\beta-\eta)]$ :

$$
I^{\alpha, \beta, \eta}\left\{z^{\gamma-1} J_{v}(z)\right\}=\frac{z^{\gamma+v-\beta-1}}{2^{v}}{ }_{2} \Psi_{3}\left[\begin{array}{c}
(\gamma+v, 2),(\gamma+\eta+v-\beta, 2) \\
(\gamma+v-\beta, 2),(\gamma+v+\alpha+\eta, 2),(v+1,1)
\end{array} \mid-\frac{1}{4} z^{2}\right] .
$$

To apply (61) from Lemma 3 , let us remind the reader again that $z^{\gamma-1} J_{v}(z)=z^{\gamma-1}{ }_{0} \Psi_{1}\left[\begin{array}{c}-- \\ (v, 1)\end{array} \mid-\frac{1}{4} z^{2}\right]$ and so, the result should be expected to appear as $a_{0+2} \Psi_{1+2}$. Alternatively, to exhibit the use of decomposition of the Saigo operator in two R-L operators (the last relation in (60)) combined with (52) from Example 2, we may proceed as follows:

$$
\begin{aligned}
R^{-\eta} z^{-\alpha-\beta} R^{\alpha+\eta}\left\{z_{0}^{\gamma-1} \Psi_{1}\left[\begin{array}{c}
-- \\
(v, 1)
\end{array} \mid-\frac{1}{4} z^{2}\right]\right\} \\
=R^{-\eta} z^{-\alpha-\beta}\left\{2 ^ { - v } z ^ { \gamma + v + \alpha + \eta - 1 } { } _ { 1 } \Psi _ { 2 } \left[\begin{array}{c}
(\gamma+v, 2) \\
\left.\left.(\gamma+v+\alpha+\eta, 2),(v+1,1) \mid-\frac{1}{4} z^{2}\right]\right\}
\end{array}\right.\right. \\
=2^{-v} R^{-\eta}\left\{z^{\gamma-\beta+\eta+v-1}{ }_{1} \Psi_{2}\left[\begin{array}{c}
(\gamma+v, 2) \\
(\gamma+v+\alpha+\eta, 2),(v+1,1)
\end{array} \mid-\frac{1}{4} z^{2}\right]\right\}=\ldots{ }_{3} \Psi_{3} \ldots, \text { as in (63). }
\end{aligned}
$$

Example 6. The more special case for Saigo fractional integral of a (weighted) cosine function is the formula from the same paper of Kilbas-Sebastian ([53], Th.5, (47)), for $\operatorname{Re} \alpha>0, \operatorname{Re} \gamma>\max [0, \operatorname{Re}(\beta-\eta)]$ :

$$
I^{\alpha, \beta, \eta}\left\{z^{\gamma-1} \cos z\right\}=\sqrt{\pi} z^{\gamma-\beta-1}{ }_{2} \Psi_{3}\left[\begin{array}{c}
(\gamma, 2),(\gamma+\eta-\beta, 2) \\
(\gamma-\beta, 2),(\gamma+\eta+\alpha, 2),\left(\frac{1}{2}, 1\right)
\end{array} \mid-\frac{1}{4} z^{2}\right] .
$$

Note that $\cos z=\sqrt{\pi z / 2} J_{-1 / 2}(z)$, and use the result (63) of Example 4 with $v=-1 / 2$. To use our general approach, we can present the cos-function as a $0 \Psi_{1}$-function, and predict the result to be $a_{0+2} \Psi_{1+2}$-function in (64).

Next, we consider a case with a more general special function, called generalized K-series. In [59] (K.) Sharma introduced an extension of both a g.h.f. $p F_{q}(z)$ and Prabhakar (three-parameter Mittag-Leffler) function $E_{\alpha, \beta}^{\gamma}(z)$ (see (8)):

$$
p \stackrel{\alpha, \beta ; \gamma}{K} q\left(a_{1}, \ldots, a_{p} ; b_{1}, \ldots, b_{q} ; z\right):=\stackrel{\alpha, \beta ; \gamma}{K}_{q}(z)=\sum_{k=0}^{\infty} \frac{\left(a_{1}\right)_{k} \ldots\left(a_{p}\right)_{k}}{\left(b_{1}\right)_{k} \ldots\left(b_{q}\right)_{k}} \frac{(\gamma)_{k} z^{k}}{\Gamma(\alpha k+\beta)}
$$

with $z, \alpha, \beta \in \mathbb{C}, \operatorname{Re} \alpha>0$, integers $p \leq q$ (and additional requirement $|z|<R=\alpha^{\alpha}$ if $p=q+1$ ). When $\gamma=1$ it reduces to the (generalized) M-series (56) by Sharma-Jain [54], Example 4. 
Example 7. Recently, Lavault [55] represented the above K-series in terms of a Wright g.h.f.:

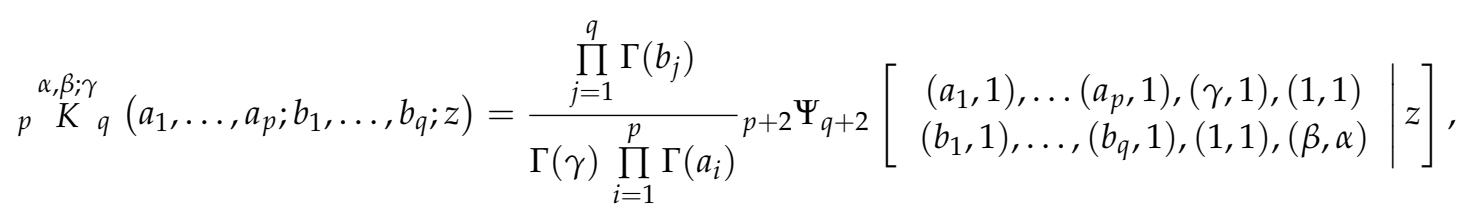

and calculated some of its FC operators, as the R-L, Saigo and M-S-M operators. As should be expected, the image of $a_{p} \stackrel{\alpha, \beta ; \gamma}{K}$-function under the $R$-L integral is $a_{p+1} \stackrel{\alpha, \beta ; \gamma}{K}{ }_{q+1}$-function (Th. 4.1 there), similarly to Example 4 for the M-series.

The Saigo operator is also derived by Lavault in [55]: for the M-series-in Th. 4.2, and for the K-series-in Cor. 4.3. Namely, Equation (4.10), [55] reads as:

$$
\left.I^{\alpha, \beta, \gamma}\left\{t^{\sigma-1} \stackrel{\xi, \eta, v}{K}_{q}\left(c z^{\mu}\right)\right\}=\frac{\prod_{1}^{q} \Gamma\left(b_{j}\right)}{\prod_{1}^{p} \Gamma\left(a_{i}\right)} \frac{z^{\sigma-\beta-1}}{\Gamma(v)} p+3 \Psi_{q+3}\left[\begin{array}{c}
\left(a_{i}, 1\right)_{1}^{p},(\sigma, \mu),(-\beta+\gamma+\sigma, \mu),(v, 1) \\
\left(b_{j}\right)_{1}^{q},(\beta+\sigma, \mu),(\alpha+\gamma+\sigma, \mu),(\eta, \xi)
\end{array}\right] c z^{\mu}\right] .
$$

Let us note that the K-series is a ${ }_{p+2} \Psi_{q+2}$-function (66), and from our Lemma 3 the expected result should be $a_{p+4} \Psi_{q+4}$-function, with indices increased by two. However, pairs of upper and lower rows' parameters appear the same and eliminate each other, therefore the result reduces to $a_{p+3} \Psi_{q+3}$, as above.

\section{Marichev-Saigo-Maeda (M-S-M) Operators of Various Special Functions}

As mentioned in Section 2.3, there is an interesting particular case of the GFC operators (20) and (23) for $m=3$, often abbreviated as M-S-M (MSM) operators. These operators have also become very popular in works dedicated to evaluate FC images of special functions. A search in Google for "Marichev-Saigo-Maeda" returns at least 2430 results, and for "MSM operator"-some 2670 results.

This operator appeared in a paper by Marichev of 1974, [39], see also in the book ([31], Section 8.4.51); and further was introduced and studied by Saigo, Saigo and Maeda in 1996, see [40], also by Saigo and Saxena $(1996,1998,2001)$, details on references are in $([6,37,58,60]$, Ch.1), etc.

For complex parameters $a, a^{\prime}, b, b^{\prime}, c, \operatorname{Re} c>0$, the Marichev-Saigo-Maeda (M-S-M) integral operator, of which the kernel is the Appel function, or Horn's function $F_{3}$ (see ([15], Vol.1), also [7])

$$
F_{3}\left(a, a^{\prime}, b, b^{\prime}, c, z, \xi\right)=\sum_{m, n=0}^{\infty} \frac{(a)_{m}\left(a^{\prime}\right)_{n}(b)_{m}\left(b^{\prime}\right)_{n}}{(c)_{m+n}} \frac{z^{m} \xi^{n}}{m ! n !}, \quad|z|<1,|\xi|<1,
$$

is defined as the linear integral operator

$$
\begin{aligned}
& I^{a, a^{\prime}, b, b^{\prime}, c} f(z)=\frac{z^{-a}}{\Gamma(c)} \int_{0}^{z}(z-\xi)^{c-1} \xi^{-a^{\prime}} F_{3}\left(a, a^{\prime}, b, b^{\prime} ; c ; 1-\frac{\xi}{z}, 1-\frac{z}{\xi}\right) f(\xi) d \xi \\
& =z^{c-a-a^{\prime}} \int_{0}^{1} \frac{(1-\sigma)^{c-1}}{\Gamma(c)} \sigma^{-a^{\prime}} F_{3}\left(a, a^{\prime}, b, b^{\prime} ; c ; 1-\sigma, 1-\frac{1}{\sigma}\right) f(z \sigma) d \sigma .
\end{aligned}
$$

Observing the representation (31) of the kernel $F_{3}$-function as a kernel of the generalized fractional integrals (20) (see Section 2.3), it is evident that the M-S-M operator is nothing but their special case 
for $m=3$. Then, in view of (21), it is also a composition of three commutable classical E-K integrals (see Kiryakova $[6,37,58])$. This fact seems to be unknown to the other authors (or is continuously ignored):

$$
\begin{aligned}
& I^{a, a^{\prime}, b, b^{\prime}, c} f(z)=z^{c-a-a^{\prime}} \int_{0}^{1} \sigma^{-a^{\prime}} G_{3,3}^{3,0}\left[\begin{array}{c}
\sigma \\
a+b, c-a^{\prime}, c-b^{\prime} \\
a, b, c-a^{\prime}-b^{\prime}
\end{array}\right] f(z \sigma) d \sigma \\
& =z^{c-a-a^{\prime}} \int_{0}^{1} G_{3,3}^{3,0}\left[\begin{array}{c}
\sigma \\
a-a^{\prime}+b, c-2 a^{\prime}, c-a^{\prime}-b^{\prime} \\
a-a^{\prime}, b-a, c-2 a^{\prime}-b^{\prime}
\end{array}\right] f(z \sigma) d \sigma \\
& =z^{c-a-a^{\prime}} \int_{0}^{1} H_{3,3}^{3,0}\left[\begin{array}{c}
\sigma \\
\left(a-a^{\prime}+b, 1\right),\left(c-2 a^{\prime}, 1\right),\left(c-a^{\prime}-b^{\prime}, 1\right) \\
\left(a-a^{\prime}, 1\right),(b-a, 1),\left(c-2 a^{\prime}-b^{\prime}, 1\right)
\end{array}\right] f(z \sigma) d \sigma \\
& =z^{c-a-a^{\prime}} I_{(1,1,1), 3}^{\left(a-a^{\prime}, b-a^{\prime}, c-2 a^{\prime}-b^{\prime}\right),\left(b, c-a^{\prime}-b, a^{\prime}\right)} f(z) \\
& =z^{c-a-a^{\prime}} I_{1}^{a-a^{\prime}, b} I_{1}^{b-a^{\prime}, c-a^{\prime}-b} I_{1}^{c-2 a^{\prime}-b^{\prime}, a^{\prime}} f(z) \text {. }
\end{aligned}
$$

The relations (31) and (69) have been recently denied and argued in the Response of authors [61] to our critical Commentary [5] to their paper [62]. Then, I needed to support (by my footnote remark to [61]) the truth of (69) as appearing also in the basic FC book by Samko-Kilbas-Marichev [31], see there Equation (10.38) (for decomposition of Saigo operator) and Equation (10.46), p. 193 (for decomposition of the M-S-M operator).

For the above reasons, to evaluate M-S-M images of special functions, which are representable as Wright g.h.f., one can use the general result of Theorem 3. Thus we have:

Lemma 4. The image of a Wright g.h.f. under the M-S-M fractional integral is given by the formula

$$
\begin{aligned}
& I^{a, a^{\prime}, b, b^{\prime}, c}\left\{z^{v}{ }_{p} \Psi_{q}\left[\begin{array}{r}
\left(a_{i}, A_{i}\right)_{1}^{p} \\
\left(b_{j}, B_{j}\right)_{1}^{q}
\end{array} \mid \lambda z^{\mu}\right]\right\} \\
& =z^{c-a-a^{\prime}}{ }_{p+3} \Psi_{q+3}\left[\begin{array}{c}
\left(a_{i}, A_{i}\right)_{1}^{p},\left(a-a^{\prime}+1+v, 1\right),\left(b-a^{\prime}+1+v, 1\right),\left(c-2 a^{\prime}-b^{\prime}+1+v, 1\right) \\
\left(b_{j}, B_{j}\right)_{1}^{q},\left(a-a^{\prime}+b+1+v, 1\right),\left(c-2 a^{\prime}+1+v, 1\right),\left(c-a^{\prime}-b^{\prime}+1+v, 1\right)
\end{array} \mid \lambda z^{\mu}\right] .
\end{aligned}
$$

The corresponding simpler result for $v=0$ is given by Corollary 4 in Kiryakova [2].

The M-S-M fractional derivatives $D^{a, a^{\prime}, b, b^{\prime}, c}$, denoted also by $I^{a, a^{\prime}, b, b^{\prime}, c}$ with $\operatorname{Re} c \leq 0$, are considered by Saigo and Maeda and by the next authors as originally defined by analogy with the Saigo derivatives $D^{\alpha, \beta, \eta}$. In view of (69), they can be considered also as special cases of the generalized fractional derivatives (23) with $m=3$, namely as $D^{a, a^{\prime}, b, b^{\prime}, c}=D_{(1,1,1), 3}^{\left(a-a^{\prime}, b-a^{\prime}, c-2 a^{\prime}-b^{\prime}\right),\left(b, c-a^{\prime}-b, a^{\prime}\right)} z^{-c}$.

The authors after Saigo-Maeda use to derive first a formula for the M-S-M image of a power function $z^{p}$, ignoring the fact that it exists in the original paper (1996) (and follows also as a particular case of our (27) in Section 2). Then, to find the M-S-M fractional integral or derivative of a particular special function, they use the standard techniques of term-by-term integration/or differentiation of the corresponding powers series. However, our general approach says that we know in advance the image of a $p \Psi_{q}$-function expected as a $p+3 \Psi_{q+3}$, see (70).

We provide a few illustrative examples for other authors' results, mentioned also in Kiryakova [2].

Example 8. The formula for the M-S-M generalized fractional integral of a weighted Bessel function:

$$
\begin{aligned}
& I^{a, a^{\prime}, b, b^{\prime}, c}\left\{z^{\gamma-1} J_{v}(z)\right\}=\frac{z^{\gamma+v-a-a^{\prime}+c-1}}{2^{v}} \\
& \times{ }_{3} \Psi_{4}\left[\begin{array}{c}
(\gamma+v, 2),\left(\gamma+v+c-a-a^{\prime}-b, 2\right),\left(\gamma+v+b^{\prime}-a^{\prime}, 2\right) \\
\left(\gamma+v+b^{\prime}, 2\right),\left(\gamma+v+c-a-a^{\prime}, 2\right),\left(\gamma+v+c-a^{\prime}-b, 2\right),(v+1,1)
\end{array} \mid-\frac{z^{2}}{4}\right],
\end{aligned}
$$


can be found in Purohit-Suthar-Kalla ([60], Th.2.1, (10)). It is supposed that $\operatorname{Rec}>0, \operatorname{Re} v>-1, \operatorname{Re}(\gamma+v)>$ $\max \left[0, \operatorname{Re}\left(a+a^{\prime}+b-c\right), \operatorname{Re}\left(a^{\prime}-b^{\prime}\right)\right]$. The same result, however, can be obtained in the way as discussed in Example 5, using the M-S-M operator's representation (69) and the result from Lemma 4. Then, by analogy with Example 6, one can derive the particular result from ([60], Cor.3.1, (24)) for the M-S-M image of $z^{\gamma-1} \cos z$, again in terms of ${ }_{3} \Psi_{4}\left(-\frac{z^{2}}{4}\right)$.

Example 9. Mondal-Nisar ([63], Th.3, (11)) evaluated the M-S-M integral (68) of the so-called generalized Bessel function

$$
W_{p, \beta, \gamma}(z)=\sum_{k=0}^{\infty} \frac{(-1)^{k} \gamma^{k}}{\Gamma\left(p+\frac{\beta}{2}+\frac{1}{2}+k\right) k !}\left(\frac{z}{2}\right)^{2 k+p} .
$$

Evidently, it is a variant (up to variable substitution) of the Bessel-Maitland-Wright function $J_{v}^{\kappa}(z)$ and of the Wright function $\phi(z)$, representable as ${ }_{0} \Psi_{1}$-function of $\left(z^{2} / 4\right)$. Then as well expected, the result comes as a ${ }_{3} \Psi_{4}$-function, since the indices are increased by 3.

Example 10. Next, in Nisar-Mondal-Agrawal ([64], Th.1), the authors derive the M-S-M operator of the Bessel-Struve function, which is representable as a $2 \times 2$-indices (multi-index, $m=2$ ) Mittag-Leffler function (9), see Examples (11) mentioned in Section 2.1, as well as a Wright g.h.f. ${ }_{1} \Psi_{1}$,

$$
S_{v}(z)=\frac{\Gamma(v)}{\sqrt{\pi}} \sum_{k=0}^{\infty} \frac{\Gamma\left(\frac{1}{2}+\frac{k}{2}\right)}{\Gamma\left(v+1+\frac{k}{2}\right)} \frac{z^{k}}{k !}=\frac{\Gamma(v)}{\sqrt{\pi}}{ }_{1} \Psi_{1}\left[\begin{array}{c}
\left(\frac{1}{2}, \frac{1}{2}\right) \\
\left(v+1, \frac{1}{2}\right)
\end{array}\right] z
$$

Then, the result for $I^{a, a^{\prime}, b, b^{\prime}, c}\left\{t^{\gamma-1} S_{v}(\lambda z)\right\}$ is expected, written in terms of $a_{1+3} \Psi_{1+3}(\lambda z)={ }_{4} \Psi_{4}(\lambda z)$, with parameters following from the general scheme.

Example 11. The M-S-M operator (68) of a generalized multi-index Mittag-Leffler function

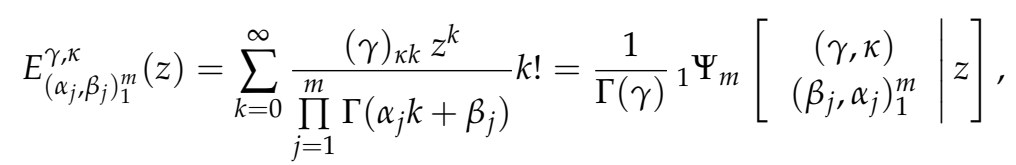

is handled in Agarwal-Rogosin-Trujillo [29]. When $m=1$ it was studied also by Srivastava-Tomovski [65]. Note that for $\gamma=\kappa=1$ the above function reduces to the (2m) multi-index Mittag-Leffler function (9). This appeared also in Saxena-Nishimoto [66] and was studied in Saxena-Pogany-Ram-Daiya [67]. The result from ([29], Th.3.1, (3.2)) is the following:

$$
\begin{aligned}
& I^{a, a^{\prime}, b, b^{\prime}, c}\left\{z^{\rho-1} E_{\left(\alpha_{j}, \beta_{j}\right)_{1}^{m}}^{\gamma, \kappa}\left(\lambda z^{\mu}\right)\right\}=\frac{z^{\rho+c-a-a^{\prime}-1}}{\Gamma(\gamma)} \\
& \times{ }_{4} \Psi_{m+3}\left[\begin{array}{c}
(\gamma, \kappa),(\rho, \mu),\left(\rho+c-a-a^{\prime}-b, \mu\right),\left(\rho+b^{\prime}-a^{\prime}, \mu\right) \\
\left(\alpha_{j}, \beta_{j}\right)_{1}^{m},\left(\rho+b^{\prime}, \mu\right),\left(\rho+c-a-a^{\prime}, \mu\right),\left(\rho+c-b-a^{\prime}, \mu\right)
\end{array} \mid \lambda z^{\mu}\right] .
\end{aligned}
$$

Using the representations of the M-S-M operator as three-tuple generalized fractional integral (69) and of this special function as $a_{1} \Psi_{m}$-function, the same formula can be evaluated by the general result in Theorem 3, that is the image is again a Wright g.h.f. but its indices are increased by three.

Example 12. We were stuck on a paper by Kumar-Gupta-Rawat [68] (very fast accepted and published with a lot of typographical problems). The authors there aim to "establish certain generalized fractional differentiation involving M-L type function with four parameters, recently introduced by Garg et al. (2016)". Namely, they have evaluated its image under the Marichev-Saigo-Maeda derivative $D^{a, a^{\prime}, b, b^{\prime}, c}$, corresponding to the integral operator (68). Their result, Theorem 1 (p.205), reads as follows:

$$
D^{a, a^{\prime}, b, b^{\prime}, c}\left\{t^{\rho-1}{ }_{\xi, \gamma} E_{\mu, v}\left(\lambda z^{\sigma}\right)\right\}=\frac{z^{\rho+a+a^{\prime}-c-1}}{\Gamma(\xi)}
$$




$$
\times{ }_{5} \Psi_{4}\left[\begin{array}{c}
(\xi, \gamma),(\rho, \sigma),\left(\rho+a+a^{\prime}+b^{\prime}-c, \sigma\right),(\rho+a-b, \sigma),(1,1) \\
(\rho-b, \sigma),\left(\rho+a+a^{\prime}-c, \sigma\right),(v, \mu),\left(\rho+a+b^{\prime}-c, \sigma\right)
\end{array} \mid \lambda z^{\sigma}\right] .
$$

The authors did not observe the fact that the considered $M-L$ type function is a case of the generalized Wright function (4), the definition of which is also given in the mentioned paper, namely:

$$
{ }_{\xi, \gamma} E_{\mu, v}(z)=\sum_{k=0}^{\infty} \frac{(\xi)_{\gamma k}}{\Gamma(\mu k+v)} z^{k}=\frac{1}{\Gamma(\xi)} \sum_{k=0}^{\infty} \frac{\Gamma(\xi+\gamma k) \Gamma(k+1)}{\Gamma(\mu k+v)} \frac{z^{k}}{k !}=\frac{1}{\Gamma(\xi)}{ }_{2} \Psi_{1}\left[\begin{array}{c}
(\xi, \gamma),(1,1) \\
(v, \mu)
\end{array} \mid z\right] .
$$

Then, its image under the M-S-M differentiation, as a three-tuple generalized fractional derivative, is well expected to be the Wright function $2+3 \Psi_{1+3}$, in view of our general results as (51) and (70).

Example 13. In [62], Agarwal-Jain-Baleanu considered the M-S-M images of the generalized Lommel-Wright function

$$
\begin{aligned}
J_{\omega, \theta}^{\varphi, m}(z) & =\left(\frac{z}{2}\right)^{\omega+2 \theta} \sum_{k=0}^{\infty} \frac{(-1)^{k}\left(\frac{z}{2}\right)^{2 k}}{(\Gamma(\theta+k+1))^{m} \Gamma(\omega+k \varphi+\theta+1)} \\
& =\left(\frac{z}{2}\right)^{\omega+2 \theta}{ }_{1} \Psi_{m+1}\left[(1,1) ;(\theta+1,1), \ldots,(\theta+1,1),(\omega+\theta+1, \varphi) ;-z^{2} / 4\right],
\end{aligned}
$$

which is a Wright g.h.f. (see Equation (1.1) there). We can note that it is also example of the multi-index M-L function (9), namely $J_{\omega, \theta}^{\varphi, m}(z)=\left(\frac{z}{2}\right)^{\omega+2 \theta}\left(\frac{z}{2}\right)^{\omega+2 \theta} E_{(1, \ldots, 1, \varphi),(\theta+1, \ldots, \theta+1, \omega+\theta+1)}^{(m+1)}\left(-\left(\frac{z}{2}\right)^{2}\right)$. Then the result, as calculated by the authors, follows directly from Theorem 3 and especially from Lemma 4 (below, $A:=$ $\chi+\omega+2 \theta, \varphi>0):$

$$
\begin{aligned}
& I_{0+}^{\xi, \xi^{\prime}, \rho, \rho^{\prime}, \varkappa}\left[t^{\chi-1} J_{\omega, \theta}^{\varphi, m}(t z)\right](x)=x^{A-\xi^{-}-\xi^{\prime}+\varkappa-1}\left(\frac{z}{2}\right)^{\omega+2 \theta} \\
& \times{ }_{4} \Psi_{4+m}\left[\begin{array}{c}
(A, 2),\left(A+\varkappa-\xi-\xi^{\prime}-\rho, 2\right),\left(A+\rho^{\prime}-\xi^{\prime}, 2\right),(1,1) \\
\left(A+\rho^{\prime}, 2\right),\left(A+\varkappa-\xi-\xi^{\prime}, 2\right),\left(A+\varkappa-\xi^{\prime}-\rho, 2\right),(\omega+\theta+1, \varphi),(\theta+1,1)
\end{array} \mid-\frac{(t z)^{2}}{4}\right],
\end{aligned}
$$

to be again a Wright g.h.f. but with indices increased by three, that is, $a_{4} \Psi_{m+4}$-function. In [62] also many special cases are derived, such as Beta-transform (that is E-K integral), Saigo operator, path integral, of the function (73) and of its particular cases. As in the Commentary [5] we discussed the possibilities to use our unified approach, the authors tried to argue with the facts in their Response [61]. The curious readers are recommended to read Kiryakova's footnote comments at the bottom to this Response [61].

\section{Multiple Gel'fond-Leontiev Operators of Multi-Index Mittag-Leffler Functions; Hyper-Bessel Operators and Functions}

We consider now GFC images of the multi-index M-L functions (9).

Lemma 5. Taking in general $m \neq n$ (m-tuple operators of GFC and $2 n$-indexed $M-L$ functions), we have

$$
\begin{aligned}
I_{\left(\beta_{k}\right), m}^{\left(\gamma_{k}\right),\left(\delta_{k}\right)}\left\{z^{c} E_{\left(\alpha_{i}\right),\left(v_{i}\right)}^{(n)}\left(\lambda z^{\mu}\right)\right\} & =I_{\left(\beta_{k}\right), m}^{\left(\gamma_{k}\right),\left(\delta_{k}\right)}\left\{z^{c}{ }_{1} \Psi_{n}\left[\begin{array}{c}
(1,1) \\
\left.\left(v_{i}, \alpha_{i}\right)_{1}^{n}\right)
\end{array} \mid \lambda z^{\mu}\right]\right\} \\
& =z^{c}{ }_{1+m} \Psi_{n+m}\left[\begin{array}{c}
(1,1),\left(\gamma_{k}+1+c / \beta_{k}, \mu / \beta_{k}\right)_{1}^{m} \\
\left(v_{i}, \alpha_{i}\right)_{1}^{n},\left(\gamma_{k}+\delta_{k}+1+c / \beta_{k}, \mu / \beta_{k}\right)_{1}^{m}
\end{array} \mid \lambda z^{m}\right] .
\end{aligned}
$$

This is an easy corollary of Theorem 3. In particular, for $c=0, \mu=1, m=n$, and for GFC parameters taken to be $\gamma_{k}=v_{k}-1, \beta_{k}=1 / \alpha_{k}, k=1,2, \ldots, m$, it happens that the parameters $\left(\gamma_{k}+1,1 / \beta_{k}\right)_{1}^{m}$ in the upper row and $\left(v_{i}, \alpha_{i}\right)_{1}^{m}$ in bottom row appear equal and cancel each other, and then the W. g.h.f. ${ }_{1+m} \Psi_{m+m}$ reduces to ${ }_{1} \Psi_{m}$, again a multi-index $M-L$ function!

Then, as proved (in other direct way) in our previous papers, we have: 
Example 14 (Kiryakova, [21,22]). For each fixed $j=1, \ldots, m$, a (classical) E-K integral of (9) reads as:

$$
I_{1 / \alpha_{j}}^{\beta_{j}-1, \delta_{j}} E_{\left(\alpha_{i}\right),\left(\beta_{i}\right)}(\lambda z)=E_{\left(\alpha_{i}\right),\left(\beta_{1}, \ldots, \beta_{j-1}, \beta_{j}+\delta_{j}, \beta_{j+1}, \ldots, \beta_{m}\right)}(\lambda z) .
$$

This is an extension of the result for an $E$-K integral (12) of an $M-L$ function: $I_{1 / \alpha}^{\beta-1, \delta} E_{\alpha, \beta}(z)=E_{\alpha, \beta+\delta}(z)$, where its second index is increased by the order of fractional integral. After m-times application of the above relation with respect to each $j=1, \ldots, m$, we obtain that a GFC integral (18) with suitably chosen parameters transforms a multi-index $M-L$ function into the same kind of multi-index $M-L$ function of which the indices of the second set are increased by the multi-order of fractional integration:

$$
I_{\left(1 / \alpha_{k}\right), m}^{\left(\beta_{k}-1\right),\left(\delta_{k}\right)} E_{\left(\alpha_{i}\right)_{1}^{m},\left(\beta_{i}\right)_{1}^{m}}(\lambda z)=E_{\left(\alpha_{i}\right)_{1}^{m},\left(\beta_{i}+\delta_{i}\right)_{1}^{m}}(\lambda z) \text {, with } \operatorname{Re} \delta_{k}>0, \gamma_{k}>-1, \alpha_{k}>0, k=1, \ldots, m, \lambda \neq 0 .
$$

If we take $\delta_{k}=\alpha_{k}, k=1, \ldots, m$, formula (77) has the form (Kiryakova, [21,22])

$$
I_{\left(1 / \alpha_{k}\right), m}^{\left(\beta_{k}-1\right),\left(\alpha_{k}\right)} E_{\left(\alpha_{i}\right),\left(\beta_{i}\right)}(\lambda z)=E_{\left(\alpha_{i}\right),\left(\beta_{i}+\alpha_{i}\right)}(\lambda z)=(\lambda z)^{-1}\left[E_{\left(\alpha_{i}\right),\left(\beta_{i}\right)}(\lambda z)-\frac{1}{\prod_{i=1}^{m} \Gamma\left(\beta_{i}\right)}\right] .
$$

According to the operational rules of the GFC (([6], Ch.5)) and rewriting the above relation for the generalized fractional derivative $D_{\left(1 / \alpha_{k}\right), m}^{\left(\beta_{k}-1-\alpha_{k}\right),\left(\alpha_{k}\right)}$ defined as in (23), we have

$$
D_{\left(1 / \alpha_{k}\right), m}^{\left(\beta_{k}-1-\alpha_{k}\right),\left(\alpha_{k}\right)} E_{\left(\alpha_{i}\right),\left(\beta_{i}\right)}(\lambda z)=(\lambda z) E_{\left(\alpha_{i}\right),\left(\beta_{i}\right)}(\lambda z)+\left[\prod_{i=1}^{m} \Gamma\left(\beta_{i}-\alpha_{i}\right)\right]^{-1} .
$$

Here one can see an analogy with the results (10.6), (10.9) from Haubold-Mathai-Saxena [17] for the R-L operators (in the case $m=1$ ).

Next, let us consider the special cases of GFC operators for which the multi-index M-L functions (9) appear as eigenfunctions, that is, these special functions are transformed into the same kind of functions with the same multi-indices.

Example 15. The so-called Gelfond-Leontiev (G-L) operators are operators of generalized integration and differentiation, defined for functions $f(z)=\sum_{j=0}^{\infty} a_{j} z_{j}$ analytic in a disk $|z|<R$, and are generated by the coefficients of a given entire function $\varphi(\sigma)$, used as a multipliers' sequence. They were introduced in a paper by Gelfond and Leontiev of 1951 (for details and references see our works, such as [6,20,21]). In the case when $\varphi(\sigma)=E_{\left(\alpha_{i}\right),\left(\beta_{i}\right)}(\sigma)$ is the multi-index M-L function (9), these operators were considered by Kiryakova [20], see also [21], etc., and called (multiple) Dzrbashjan-Gelfond-Leontiev (D-G-L) operators. We defined them as follows:

$$
\begin{aligned}
\mathfrak{D} f(z) & =\sum_{j=1}^{\infty} a_{j} \frac{\Gamma\left(\beta_{1}+j \alpha_{1}\right) \ldots \Gamma\left(\beta_{m}+j \alpha_{m}\right)}{\Gamma\left(\beta_{1}+(j-1) \alpha_{1}\right) \ldots \Gamma\left(\beta_{m}+(j-1) \alpha_{m}\right)} z^{j-1}, \\
\mathfrak{L} f(z) & =\sum_{j=0}^{\infty} a_{j} \frac{\Gamma\left(\beta_{1}+j \alpha_{1}\right) \ldots \Gamma\left(\beta_{m}+j \alpha_{m}\right)}{\Gamma\left(\beta_{1}+(j+1) \alpha_{1}\right) \ldots \Gamma\left(\beta_{m}+(j+1) \alpha_{m}\right)} z^{j+1},
\end{aligned}
$$

and noted that the image functions $\mathfrak{D} f(z), \mathfrak{L} f(z)$ are also analytic functions in the same disk $|z|<R$.

We have shown (e.g., in [21,22]) that the operators (78) can be analytically extended (outside a disk, to holomorphic functions in starlike domain) to operators of GFC, namely to generalized integrals and derivatives of fractional multi-order $\left(\alpha_{1}, \ldots, \alpha_{m}\right)$ as follows:

$$
\mathfrak{L} f(z)=z^{1} I_{\left(1 / \alpha_{k}\right), m}^{\left(\beta_{k}-1\right),\left(\alpha_{k}\right)} f(z), \mathfrak{D} f(z)=z^{-1} D_{\left(1 / \alpha_{k}\right), m}^{\left(\beta_{k}-1-\alpha_{k}\right),\left(\alpha_{k}\right)} f(z)-z^{-1} f(0)\left[\prod_{k=1}^{m} \frac{\Gamma\left(\beta_{k}\right)}{\Gamma\left(\beta_{k}-\alpha_{k}\right)}\right] .
$$


Then, as proved in [21,22], etc., and seen also in the end of Example 14, the multi-index M-L function (9) is a solution of the differential equation of fractional multi-order

$$
\mathfrak{D} E_{\left(\alpha_{i}\right),\left(\beta_{i}\right)}(\lambda z)=E_{\left(\alpha_{i}\right),\left(\beta_{i}\right)}(\lambda z) \text {, that is, } E_{\left(\alpha_{i}\right),\left(\beta_{i}\right)}(z) \text { is an eigenfunction of the operator } \mathfrak{D} \text {. }
$$

In view of this relation, the multi-index $M$-L function serves as an eigenfunction of the $D$-G- $L$ differentiation generated by its own coefficients!

In the case of the 3m-parametric M-L type functions (multi-index Prabhakar functions, [26])

$E_{\left(\alpha_{i}\right),\left(\beta_{i}\right)}^{\left(\gamma_{i}\right), m}(z)=\sum_{k=0}^{\infty} \frac{\left(\gamma_{1}\right)_{k} \ldots\left(\gamma_{m}\right)_{k}}{\Gamma\left(\alpha_{1} k+\beta_{1}\right) \ldots \Gamma\left(\alpha_{m} k+\beta_{m}\right)} \frac{z^{k}}{(k !)^{m}}$, with Pochhamer symbols $\left(\gamma_{i}\right)_{k}:=\frac{\Gamma\left(\gamma_{i}+k\right)}{\Gamma\left(\gamma_{i}\right)}$

the R-L, classical E-K operators and some multiple E-K operators are evaluated in the works of Paneva-Konovska, for example, the book [19].

Example 16. In 1966, and his later works, Dimovski [41] introduced a very general class of differential operators of arbitrary (integer) order generalizing the Bessel operators of second order. His aims were to develop operational calculus for these operators, both via a Laplace-type integral transform and by the Mikusinski algebraical approach. These operators have the alternative representations

$$
B f(t)=t^{\alpha_{0}} \frac{d}{d t} t^{\alpha_{1}} \frac{d}{d t} \cdots t^{\alpha_{m-1}} \frac{d}{d t} t^{\alpha_{m}} f(t)=t^{-\beta} P_{m}\left(t \frac{d}{d t}\right) f(t)=t^{-\beta} \prod_{k=1}^{m}\left(t \frac{d}{d t}+\beta \gamma_{k}\right) f(t), t>0,
$$

with arbitrary parameters $\alpha_{0} ; \alpha_{k}, \gamma_{k}, k=1, \ldots, m ; \beta>0 ; P_{m}$ a polynomial of degree $m$, and their different cases were studied by many authors as appearing in various equations of mathematical physics, problems in analysis, etc., disciplines. The name "hyper-Bessel differential operator" for (80) was introduced by Kiryakova in the further studies on the topic, for example ([6], Ch.3), and next ones as [69]. For the linear right inverse operator denoted by $L$ and called hyper-Bessel integral operator (such that $B L f(t)=f(t)$ ), we have found a representation by an integral operator with Meijer's $G_{m, m}^{m, 0}$-function in the kernel, and later, the same kind of integral representation also for its fractional powers $L^{\lambda}, \lambda>0$. These results were the hint of how to introduce the operators of GFC: the generalized integration and differentiation (20) and (26) of arbitrary fractional multi-order $\left(\delta_{1}, \delta_{2}, \ldots, \delta_{m}\right)$ instead of the multi-order $(\lambda, \lambda, \ldots, \lambda)$ for $L^{\lambda}$. The story is explained in [69]. Due to the representation of the hyper-Bessel operators in the form: $B=t^{-1} D_{(\beta, \beta, \ldots, \beta), m}^{\left(\gamma_{k}-1\right),(1,1, \ldots, 1)}, L=t I_{(\beta, \beta, \ldots, \beta), m}^{\left(\gamma_{k}\right),(1,1, \ldots, 1)}$, these operators are important examples of the generalized "fractional" derivatives and also of the Gelfond-Leontiev operators (78). Indeed, for simplicity we take $\beta=1$ and $\gamma_{m}=0$, then we have that $B=t^{-1} D_{(1)_{1}^{m}, m}^{\left(\gamma_{k}-1\right)_{1}^{m},(1)_{1}^{m}}$ is a particular case of the operator denoted by $\mathfrak{D}$ in the previous Example 15, with modified denotations.

Consider the $m$-th order (that is, of multi-order $(1,1, \ldots, 1)$ ) hyper-Bessel differential equation

$$
B y(t)=\lambda y(t), \quad \lambda \neq 0 .
$$

In ([6], Ch.3, Th.3.4.3) and the next corollaries (see also [37]), we proved that the functions, $j=1, \ldots, m$ :

$$
\begin{aligned}
& y_{j}(t)=G_{0, m}^{1,0}\left[-\lambda t \mid \begin{array}{c}
-- \\
-\gamma_{j},-\gamma_{1}, \ldots,-\gamma_{j-1},-\gamma_{j+1}, \ldots,-\gamma_{m-1}, 0
\end{array}\right] \\
& =\frac{(\lambda t)^{-\gamma_{j}}}{\prod_{k=1}^{m} \Gamma\left(\gamma_{k}+1\right)}{ }_{0} F_{m-1}\left(\left(1+\gamma_{i}-\gamma_{j}\right)_{i \neq j} ; \lambda t\right):=J_{\left(1+\gamma_{i}-\gamma_{j}\right)_{i \neq j}}^{(m-1)}(\lambda t), \text { the hyper-Bessel functions, }
\end{aligned}
$$


form a fundamental system of solutions of equation (81) in a neighborhood of origin $t=+0$. Under the assumptions of Th.3.4.3 in [6], $\gamma_{1}<\ldots<\gamma_{m}<\gamma_{1}+1$ and $\gamma_{m}=0$, we have that $-\gamma_{j} \in(0,1)$ for all $j=1, \ldots, m$, and one of these solutions, for $j=m$, can be written as

$$
\begin{aligned}
y_{m}(t) & =\left[\prod_{k=1}^{m}\left(\gamma_{k}+1\right)\right]_{0}^{-1}{ }_{0} F_{m-1}\left(\left(1+\gamma_{i}\right)_{1}^{m-1} ; \lambda t\right)=\left[\prod_{k=1}^{m}\left(\gamma_{k}+1\right)\right]^{-1}{ }_{1} F_{m}\left(1 ;\left(1+\gamma_{i}\right)_{1}^{m-1}, 1 ; \lambda t\right) \\
& ={ }_{1} \Psi_{m}\left[\begin{array}{c}
(1,1) \\
\left(1+\gamma_{i}\right)_{1}^{m-1}, 1
\end{array} \mid \lambda z\right]=E_{(1,1, \ldots, 1),\left(1+\gamma_{i}\right)_{1}^{m}}(\lambda z), \text { a case of the multi-index } M-L \text { functions. }
\end{aligned}
$$

Therefore, Example 16 appears a special case of Example 15, and shows that the multi-index Mittag-Leffler functions (9) can be seen also as "fractional indices" analogs, extensions of the hyper-Bessel functions (82), which themselves are multi-index variants of the classical Bessel function.

\section{Some "New" Special Functions and Their FC Images}

Recently, some authors claimed to introduce and consider "new" SF. Among these are examples of the so-called $k$-analogs of the Bessel and Mittag-Leffler functions, some generalized multi-index Bessel and Mittag-Leffer functions, and some $S$-functions. The mentioned $k$-analogs are based on the use of the $k$ - $\Gamma$-function, which, however, can be rewritten in terms of the "classical" $\Gamma$-function:

$$
\Gamma_{k}(s)=\int_{0}^{\infty} \exp \left(-\frac{t^{k}}{k}\right) t^{s-1} d t=k^{\frac{s}{k}-1} \Gamma\left(\frac{s}{k}\right), \quad s \in \mathbb{C}, \operatorname{Re}(s)>0, k>0 ; \Gamma(.) \text { the Gamma-function. }
$$

Usually, the denotations include also the k-analogs of the Pochhamer symbol:

$$
(\eta)_{v, \kappa}:=\Gamma_{k}(\eta+v \kappa) / \Gamma_{k}(\lambda), \quad \eta \in \mathbb{C} \backslash\{0\}, \nu \in \mathbb{C},
$$

and in view of (83) are representable again by means of classical Gamma-functions.

Then, one can easily observe that such "new SF" are just cases of the Wright generalized hypergeometric function ${ }_{p} \Psi_{q}$. Therefore, all the results provided by the mentioned authors to evaluate FC operators of these special functions follow from our general ones, say from Theorems 3 and 4 , or the special cases as Lemmas 1 and 2 (for E-K operators, incl. R-L ones), Lemma 3 (for Saigo operators), Lemma 4 (for M-S-M operators), and so on. As an illustration, we repeat some examples from Kiryakova [4].

Example 17. A generalization of the Bessel function, called generalized $k$-Bessel function was introduced by Gehlot [70] and studied by Mondal [71], Shaktawat et al. [72], defined as

$$
W_{v, c}^{k}(z)=\sum_{n=0}^{\infty} \frac{(-c)^{n}}{\Gamma_{k}(n k+v+k)} \cdot \frac{(z / 2)^{2 n+\frac{v}{k}}}{n !}, z \in \mathbb{C}, k>0, \operatorname{Re}(v)>-1, c \in \mathbb{C} .
$$

Lets us note that this function is practically a Wright g.h.f. ${ }_{0} \Psi_{1}$, and even a simpler g.h.f. ${ }_{0} F_{1}$ of the same type as the classical Bessel function:

$$
\begin{aligned}
& W_{v, c}^{k}(z)=(z / 2)^{v / k} \sum_{n=0}^{\infty} \frac{\left[-c(z / 2)^{2}\right]^{n}}{k^{n+1+(v / k)} \Gamma(n+1+(v / k)) \Gamma(n+1)}=\frac{(z / 2)^{v / k}}{k^{1+(v / k)}} \sum_{n=0}^{\infty} \frac{\left[-c(z / 2)^{2}\right]^{n}}{k^{n} \Gamma(1+(v / k)+n .1) \Gamma(1+n .1)} \\
& =\frac{(z / 2)^{v / k}}{k^{1+(v / k)}} \sum_{n=0}^{\infty} \frac{\left[-(c / k)(z / 2)^{2}\right]^{n}}{\Gamma(1+(v / k)+n .1) \Gamma(1+n .1)}=\frac{(z / 2)^{v / k}}{k^{1+(v / k)}}{ }_{1} \Psi_{2}\left[\begin{array}{c}
(1,1) \\
\left(1+\frac{v}{k}, 1\right),(1,1)
\end{array} \mid-\frac{c}{k}\left(\frac{z}{2}\right)^{2}\right] \\
& =\frac{(z / 2)^{v / k}}{k^{1+(v / k)}}{ }_{0} \Psi_{1}\left[\begin{array}{c}
-- \\
\left(1+\frac{v}{k}, 1\right)
\end{array} \mid-\frac{c}{k}\left(\frac{z}{2}\right)^{2}\right]=\frac{(z / 2)^{v / k}}{k^{1+(v / k)} \Gamma(1+v)}{ }_{0} F_{1}\left(-; 1+\frac{v}{k} ;-\frac{c}{k} \frac{z^{2}}{4}\right) \text {. }
\end{aligned}
$$


Naturally, for $k=1, c=1$, (85) becomes the classical Bessel function:

$$
W_{v, 1}^{1}(z)=\frac{(z / 2)^{v}}{\Gamma(1+v)}{ }_{0} F_{1}\left(-; 1+v ;-\frac{z^{2}}{4}\right)
$$

In the case $c=1$, Gehlot [70] considered (85) as a solution of a $k$-Bessel differential equation. Mondal [71] studied its properties for complex $c \in \mathbb{C}$. Shaktawat et al. [72] evaluated the M-S-M operators of FC of this function. In view of Lemma 4, the result there (Th.1, (18)) is well expected to appear in terms of the ${ }_{3} \Psi_{4}$-function (because the 3-tuple FC integral increases by three the indices of the initial ${ }_{0} \Psi_{1}$-function).

Example 18. The simplest $k$-analogs of the M-L function are considered by Dorrego-Cerruti [73] and Gupta and Parihar [74], and very recently (2020/2021) studied also by Ali et al. [47]:

$$
E_{k, \alpha, \beta}(z)=\sum_{n=0}^{\infty} \frac{z^{n}}{\Gamma_{k}(\alpha n+\beta)}, \quad \operatorname{resp} . \quad E_{k ; v, \rho}^{\delta}=\sum_{n=0}^{\infty} \frac{(\delta)_{\eta, k} z^{n}}{\Gamma_{k}(v n+\rho) n !} .
$$

Various further extensions appeared, as the generalized k-Mittag-Leffler function, studied by Gupta and Parihar [74] and Nisar-Eata-Dhaifalla-Choi [75] in the form (note that the index $p$ was missing in these authors' original denotation):

$$
E_{\kappa, \alpha, \beta}^{\eta, \delta, p, q}(z):=\sum_{n=0}^{\infty} \frac{(\eta)_{q n, \kappa}}{\Gamma_{k}(\alpha n+\beta)(\delta)_{p n, \kappa}} z^{n}, \quad \kappa, p, q \in \mathbb{R}_{+} ; \alpha, \beta, \eta, \delta \in \mathbb{C},
$$

with $\min \{\operatorname{Re}(\alpha), \operatorname{Re}(\beta), \operatorname{Re}(\eta), \operatorname{Re}(\delta)\}>0 ; q \leq \operatorname{Re}(\alpha)+p ;$ the $k$-Pochhammer symbol as in (84). Again, the function (87) can be rewritten as a Wright g.h.f., now as ${ }_{2} \Psi_{2}$. Using the representations for (83) and (84) we have, respectively:

$$
\begin{gathered}
(\eta)_{q n, \kappa}=\ldots=k^{q n \kappa / k} \Gamma\left(\frac{\eta+q n \kappa}{k}\right) / \Gamma\left(\frac{\eta}{k}\right) ;(\delta)_{p n, \kappa}=\ldots=k^{p n \kappa / k} \Gamma\left(\frac{\delta+p n \kappa}{k}\right) / \Gamma\left(\frac{\delta}{k}\right) ; \\
\text { and } \Gamma_{k}(\alpha n+\beta)=k^{\alpha n+\beta / k} \cdot k^{-1} \Gamma\left(\frac{\alpha n+\beta}{k}\right) .
\end{gathered}
$$

Then,

$$
\begin{aligned}
E_{\kappa, \alpha, \beta}^{\eta, \delta, p, q}(z) & =k^{1-\frac{\beta}{k}} \frac{\Gamma(\delta / k)}{\Gamma(\eta / k)} \sum_{n=0}^{\infty} \frac{\Gamma\left(\frac{\eta}{k}+n \cdot \frac{q \kappa}{k}\right) \Gamma(1+n .1)}{\Gamma\left(\frac{\delta}{k}+n \cdot \frac{p \kappa}{k}\right) \Gamma\left(\frac{\beta}{k}+n \cdot \frac{\alpha}{k}\right)} \cdot \frac{\left[k^{\frac{q \kappa-p \kappa-\alpha}{k}} z\right]^{n}}{n !} \\
& =k^{1-\frac{\beta}{k}} \frac{\Gamma(\delta / k)}{\Gamma(\eta / k)} 2_{2} \Psi_{2}\left[\begin{array}{c}
\left(\frac{\eta}{k}, \frac{q \kappa}{k}\right),(1,1) \\
\left(\frac{\delta}{k}, \frac{p \kappa}{k}\right),\left(\frac{\beta}{k}, \frac{\alpha}{k}\right)
\end{array} \mid k^{\frac{(q-p) \kappa-\alpha}{k}} z\right] .
\end{aligned}
$$

By the standard techniques, Nisar-Eata-Dhaifalla-Choi [75] evaluated FC operators of the functions (87). In view of our general results, as expected, the results are ${ }_{5} \Psi_{5}$-functions (for the MSM operators, Ths. 1-2, 3-4) there), and in particular, ${ }_{4} \Psi_{4}$-functions (for the Saigo operators, Cor. 3.1-3.2, there). Also, the pathway integrals (that are related to E-K integrals) are calculated.

Example 19. The so-called multi-index Bessel function:

$$
J_{\left(\beta_{j}\right)_{m}, \kappa, b}^{\left(\alpha_{j}\right)_{m}, \gamma, c}(z)=\sum_{k=0}^{\infty} \frac{c^{k}(\gamma)_{k k}}{\prod_{j=1}^{m} \Gamma\left(\alpha_{j} k+\beta_{j}+\frac{b+1}{2}\right)} \cdot \frac{z^{k}}{k !}, \quad m=1,2,3, \ldots,
$$

with the Pochhammer symbol $(\gamma)_{\kappa k}$, were introduced and studied in a series of papers by Nisar at al., see, for example, Nisar-Purohit-Parmar [76]. The authors proposed a result for the $R$-L fractional integral of (88), 
unfortunately written wrongly in their Theorem 1, Equation (2.4) of [76] as $a_{2} \Psi_{2}$-function, although it is evidently $a_{2} \Psi_{m+1}$ function having $(m+1)$ parameters in the low row. The true result should be

$$
R^{\lambda}\left\{t^{\delta-1} J_{\left(\beta_{j}\right)_{m}, \varkappa, b}^{\left(\alpha_{j}\right)_{m}, c}(z)\right\}=\frac{1}{\Gamma(\gamma)} z^{\delta+\lambda-1}{ }_{2} \Psi_{m+1}\left[\begin{array}{c}
(\gamma, \varkappa),(\delta, 1) \\
\left(\beta_{j}+\frac{b+1}{2}, \alpha_{j}\right)_{1}^{m},(\lambda+\delta, 1)
\end{array} ; c z\right]
$$

However, it is easily seen that (88) is: $J_{\left(\beta_{j}\right)_{m}, \kappa, b}^{\left(\alpha_{j}\right)_{m}, \gamma, c}(z)=\frac{1}{\Gamma(\gamma}{ }_{1} \Psi_{m}\left[\begin{array}{c}(\gamma, \varkappa) \\ \left(\beta_{j}+\frac{b+1}{2}, \alpha_{j}\right)_{j=1}^{m} ; c z\end{array}\right]$, so this result follows directly from our Lemma 1. Note that the function (88) is also a special case of the generalized multi-index $M-L$ function in Example 11 with $\beta_{j} \mapsto \beta_{j}+(b+1) / 2$, and then the results for its images under FC operators follow from these in Agarwal-Rogosin-Trujillo [29].

Very recently (published 24 September 2020), in Mubeen etal. [48], the authors considered integral transforms, including FC operators, of yet more general SF called "extended generalized multi-index Bessel function" introduced by Kamarujjama-Khan-Khan (2019) with an additional member $(\delta)_{k}$ in the denominator of the series, as:

$$
J_{\left(\beta_{j}\right)_{m, k, b, \delta}}^{\left(\alpha_{j}\right)_{m}, \gamma, c}(z)=\sum_{k=0}^{\infty} \frac{(\gamma)_{k k}(-c z)^{k}}{(\delta)_{k} \prod_{j=1}^{m} \Gamma\left(\alpha_{j} k+\beta_{j}+\frac{b+1}{2}\right)} .
$$

Following similar manipulations as we did in [4] (Section 5.3, Equation (48)) for the case of (88), one can show that

$$
J_{\left(\beta_{j}\right)_{m}, \kappa, b, \delta}^{\left(\alpha_{j}\right)_{m}, \gamma, c}(z)=\frac{\Gamma(\delta)}{\Gamma(\gamma)} 2 \Psi_{m+1}\left[\begin{array}{c}
(\gamma, \kappa),(1,1) \\
(\delta, 1),\left(\beta_{j}+\frac{b+1}{2}, \alpha_{j}\right)_{1}^{m}
\end{array} \mid-c z\right]
$$

and is evidently reducible to (88) for $\delta=1$. Therefore, the M-S-M fractional integral will be a ${ }_{5} \Psi_{m+4}$-function-presented as an explicit SF, instead of the authors' hardly visible result in form of some unknown complicated series, compared with Th.5.3, [48] for the extension (1.17) of the function (90).

Example 20. The S-function was introduced in Saxena-Daiya [77] as a "new" special function extending the M-L function ( $p=q=0, k=1)$, the Prabhakar function (8), the M-series (56) of Sharma and Jain [54] $(\gamma=1$, $k=1)$, etc., by

$$
S[z]:=S_{(p, q)}^{\alpha, \beta, \gamma, \tau, k}\left(a_{1}, \ldots, a_{p} ; b_{1}, \ldots, b_{q} ; z\right)=\sum_{n=0}^{\infty} \frac{\left(a_{1}\right)_{n} \ldots\left(a_{p}\right)_{n} \cdot(\gamma)_{n \tau, k}}{\left(b_{1}\right)_{n} \ldots\left(b_{q}\right)_{n} \cdot \Gamma_{k}(n \alpha+\beta)} \cdot \frac{z^{n}}{n !},
$$

with $k \in \mathbb{R}, ; \alpha, \beta, \gamma, \tau \in \mathbb{C} ; \operatorname{Re}(\alpha)>0 ; \operatorname{Re}(\alpha)>k, \operatorname{Re}(\tau), p<q+1$. For $p=q=0$ it reduces to the generalized $k$-Mittag-Leffler function $E_{k, \alpha, \beta}^{\gamma, \tau}(z)$, see in Example 18, the simplest case by Gupta and Parihar [74].

However, as shown in Kiryakova ([4], Section 5.4), this S-function (91) appears to be a Wright g.h.f. (4) of the form ${ }_{p+1} \Psi_{q+1}\left(z k^{\tau-\frac{\alpha}{k}}\right)$, namely:

$$
S[z]=k^{1-\frac{\beta}{k}} \frac{\Gamma\left(b_{1}\right) \ldots \Gamma\left(b_{q}\right)}{\Gamma\left(a_{1}\right) \ldots \Gamma\left(a_{p}\right) \cdot \Gamma\left(\frac{\gamma}{k}\right)} p+1 \Psi_{q+1}\left[\begin{array}{c}
\left(a_{1}, 1\right), \ldots,\left(a_{p}, 1\right),\left(\frac{\gamma}{k}, \tau\right) \\
\left(b_{1}, 1\right), \ldots,\left(b_{q}, 1\right),\left(\frac{\beta}{k}, \frac{\alpha}{k}\right)
\end{array} ; z k^{\tau-\frac{\alpha}{k}}\right] .
$$

Unfortunately, this fact has not been observed neither by the authors of [77] introducing it, nor by their numerous followers. Then, all results for images of FC operators, such as $R-L, E-K$, Saigo, $M-S-M$, the Euler-transform (which is in fact E-K operator), Laplace transform, follow as images of the Wright function according to our general results, say Theorem 4.1. Then, as evaluated in [77], Th. 2.10, (32), the Euler transform is a function ${ }_{p+2} \Psi_{q+2}\left(z k^{\tau-\frac{\alpha}{k}}\right)$, because the indices are increased by one for the E-Koperator; the Saigo operators will increase the indices by two; the M-S-M integral will be in terms of Wright function with indices increased by three, namely: $(p+1)+3 \Psi_{(q+1)+3}$, etc.

Special cases of (91) are the generalized K-series (65) and M-series (56), see Examples 4 and 7. 
Example 21. The generalized $k$-Wright function (multi-parametric $k-M-L$ function) is introduced by Purohit and Badguzer [78] as a k-extension of the Wright g.h.f. (4):

$$
{ }_{p} \Psi_{q}^{k}(z)={ }_{p} \Psi_{q}^{k}\left[\begin{array}{c}
\left(a_{1}, A_{1}\right), \ldots,\left(a_{p}, A_{p}\right) \\
\left(b_{1}, B_{1}\right), \ldots,\left(b_{q}, B_{q}\right)
\end{array} \mid z\right]=\sum_{n=0}^{\infty} \frac{\Gamma_{k}\left(a_{1}+n A_{1}\right) \ldots \Gamma_{k}\left(a_{p}+n A_{p}\right)}{\Gamma_{k}\left(b_{1}+n B_{1}\right) \ldots \Gamma_{k}\left(b_{q}+n B_{q}\right)} \frac{z^{n}}{n !}, \quad k>0 .
$$

However, from the representation (83), it is seen that this "new" function is again a Wright generalized hypergeometric function, namely:

$$
\text { const }_{p+1} \Psi_{q+1}\left[\begin{array}{c}
\left(a_{i} / k, A_{i} / k\right)_{i=1}^{p} \\
\left(b_{j} / k, B_{j} / k\right)_{j=1}^{q}
\end{array} \mid k^{\left(A_{1}+\ldots+A_{p}-B_{1}-\ldots-B_{q}\right) / k} \cdot z\right] .
$$

Then the M-S-M operators evaluated for (92) by these authors can appear directly from our general results (say, Lemma 4) in terms of $p+4 \Psi_{q+4}$-functions.

\section{Conclusions}

10.1. The researchers on the topic can be advised to follow a procedure like this:

(1) Check if the considered SF can be presented as a Wright g.h.f. ${ }_{p} \Psi_{q}$ or as simpler ${ }_{p} F_{q}$-function; in more complicated cases, or if it is a Fox $H$-function or a Meijer $G$-function;

(2) Check if the operator of FC to be evaluated is some special case of the GFC operators, that is, if it can be presented as a composition of classical R-L or E-K operators (also in the form (20) and (26));

(3) Then, apply a general result like Theorem 3, Theorem 4 (or more generally, Theorem 2) and their special cases (Lemmas 1-4) and the examples, provided in this survey.

10.2. In Section 3 we first give the images of the $S F$ (the generalized hypergeometric functions ${ }_{p} \Psi_{q}$, ${ }_{p} F_{q}$ and their simplest cases) for the classical FC operators: E-K and R-L, and show that these are the same kinds of functions of which the indices $p, q$ are increased by 1 . Then, the images under the GFC operators are obtained by $m$-times application of these results, in Section 4 . Our result states that the image of $a_{p} \Psi_{q}$-function (resp. $p F_{q}$-function) under any (m-tuple) GFC operator can be predicted by Theorems 3 and 4 to be $a_{p+m} \Psi_{q+m}$-function (resp. $p+m F_{q+m}$-function) with additional parameters depending on those of the FC operators. Using this general approach, one can avoid application of the standard term-by-term integration/differentiation of the power series for each particular special function the authors choose to treat.

10.3. For the proofs of Theorems 3 and 4 and their corollaries, see Kiryakova [2-4], and for their alternative interpretations - in other our works as ([6,22,24,50], Ch.4), [27]. The basic idea is that by means of a multiple ( $m$-tuple) operator of GFC each $p+m \Psi_{q+m}$-function (resp. a $p+m F_{q+m}$-function) can be reduced to a $p \Psi_{q}$-function (resp. a $p F_{q}$-function), see comments and formula (50) before Theorem 4. Thus, by a suitable number of steps, from any ${ }_{p} \Psi_{q}$-function (resp. ${ }_{p} F_{q}$-function) we can reach to one of the three basic generalized hypergeometric functions, depending on either $p<q, p=q$ or $p=q+1:{ }_{0} \Psi_{q-p, 1} \Psi_{1,2} \Psi_{1}$ (resp. ${ }_{0} F_{q-p, 1} F_{1,2} F_{1}$ ). Additionally, by an Erdélyi-Kober operator these are reducible to one of the three elementary functions: $\cos z, z^{\alpha} \exp (z)$ or $(1-z)^{\alpha} z^{\beta}$. Details are given in Kiryakova [27], submitted to this Journal. As a conclusion, we have classified the g.h.f., that is the SF, in three basic classes: "g.h.f. of Bessel/cosine type", "g.h.f. of confluent/exp type" and "g.h.f. of Gauss/beta-distribution type", each of these classes with own specific properties. Thus, the title of Kiryakova [50] appeared as: "All the special functions are fractional differintegrals of elementary functions".

10.4. In some papers, the authors evaluate an operator of FC of a particular special function in terms of another special function. Or even, the final result is written only as a series not recognized as some SF. However, for both theoretical reasons and possible applications, the results can be useful when a GFC operator transforms a special function from some class into a special function of the same class, although with changed (increased/decreased) indices and additional parameters. Such are 
our Theorems 3 and 4 , showing that a ${ }_{p} \Psi_{q}$-function (resp. a $F_{q}$-function) is transformed into a ${ }_{p+m} \Psi_{q+m}$-function (resp. a $p+m F_{q+m}$-function). We discussed similar results also for some particular cases of SF, such as for the (classical) Wright function $\phi(\alpha, \beta, z)$, the $M$-series $p M_{q}(z)$. Among the illustrative examples for SF having FC images of the same class, are our formulas: (32), (36), (37), (39), (40), (41), (46), (49), (51), and their corollaries like (55)-(58),(61), (62), (70), (77).

10.5. Next goal: the most useful results on the topic are when we can specify an operator of GFC corresponding to the considered special function, so that this function is to be its eigenfunction. In this survey we give such examples, say for the multi-index Mittag-Leffler functions $E_{\left(\alpha_{i}\right),\left(\beta_{i}\right)}(z)$ and the hyper-Bessel functions of Delerue ${ }_{0} F_{m-1}\left(\left(1+\gamma_{i}\right)_{1}^{m-1} ; z\right)$. These are the formulas (79), (81) and (82), etc. Another example, for the eigenfunction of the simplest fractional order differential equation, is the Rabotnov function $z^{\alpha-1} E_{\alpha, \alpha}\left(z^{\alpha}\right)$ (called also fractional exponent), namely:

$$
D^{\alpha} y_{\alpha}(z)=\lambda y_{\alpha}(z) \text {, where } y_{\alpha}(z)=z^{\alpha-1} E_{\alpha, \alpha}\left(\lambda z^{\alpha}\right), \quad \alpha>0, \lambda \neq 0
$$

10.6. Many authors are publishing results on the images of particular special functions under some integral transforms like the Laplace transform, Mellin transform, Euler (Beta) transform, Whittaker transform. Observe that the Euler transform (called so after the Euler integral formula for the Gauss function) is just a case of the Erdélyi-Kober fractional integral (12), as an extension of the Riemann-Liuoville fractional integral (14). Therefore, there is no need to separately evaluate these two transforms (Euler transform and Riemann-Liouville operator), and what is more, to repeat such calculations for each particular special function. One can just apply the general result, as in Lemma 1. Note that the so-called pathway-transform is also closely related to the E-K integral. To evaluate a Laplace transform, say for any special function which is an $H$-function, one can use the general integral formula (44) and the representation of the kernel exponential function as a Wright g.h.f. (see (42), Example 1), then also as a $H$-function: $\exp (-z)=H_{0,1}^{1,0}\left[\begin{array}{c}-- \\ (0,1)\end{array}\right]$. As already mentioned in Remark 1, a basic approach to evaluate integral transforms (also FC operators) of special functions relies on their images under the Mellin transform in terms of Gamma-functions, to which the fundamental book by Marichev [8] is devoted.

Author Contributions: The ideas and results in this paper survey and reflect the author's (V. K.) sole contributions, resulting from more than 30 years of research on the topic. The author haves read and agreed to the published version of the manuscript.

Funding: This research received no financial funding.

Acknowledgments: This paper is done under the working programs on bilateral collaboration contracts of Bulgarian Academy of Sciences with Serbian and Macedonian Academies of Sciences and Arts, and under the COST program, COST Action CA15225 'Fractional'.

Conflicts of Interest: The author declares no conflict of interest.

\section{References}

1. Machado, J.A.T.; Kiryakova, V. Recent history of the fractional calculus: Data and statistics. In Handbook of Fractional Calculus with Applications. Volume 1: Basic Theory; Kochubei, A., Luchko, Y., Eds.; De Gryuter: Berlin, Germany, 2019; pp. 1-21. [CrossRef]

2. Kiryakova, V. Fractional calculus operators of special functions?-The result is well predictable! Chaos Solitons Fractals 2017, 102, 2-15. [CrossRef]

3. Kiryakova, V. Use of fractional calculus to evaluate some improper integrals of special functions. AIP Conf. Proc. 2017, 1910, 050012. [CrossRef]

4. Kiryakova, V. Fractional calculus of some "new" but not new special functions: $k$-, multi-index-, and S-analogues. AIP Conf. Proc. 2019, 2172, 0500088. [CrossRef] 
5. Kiryakova, V. Commentary: "A remark on the fractional integral operators and the image formulas of generalized Lommel-Wright function". Front. Phys. 2019, 7, 145. [CrossRef]

6. Kiryakova, V. Generalized Fractional Calculus and Applications; Longman-J. Wiley: Harlow, UK; New York, NY, USA, 1994.

7. Prudnikov, A.P.; Brychkov, Y.; Marichev, O.I. Integrals and Series, Vol. 3: More Special Functions; Gordon and Breach Sci. Publ.: New York, NY, USA; London, UK; Paris, France; Tokyo, Japan, 1992.

8. Marichev, O.I. Handbook of Integral Transforms of Higher Transcendental Functions, Theory and Algorithmic Tables; Ellis Horwood: Chichester, UK, 1983; Translated from Russian; Method of Evaluation of Integrals of Special Functions (In Russian); Nauka i Teknika, Minsk, Belarus, 1978.

9. Srivastava, H.M.; Gupta, K.S.; Goyal, S.P. The H-Functions of One and Two Variables with Applications; South Asian Publs: New Delhi, India, 1982.

10. Kilbas, A.A.; Srivastava, H.M.; Trujillo, J.J. Theory and Applications of Fractional Differential Equations; Elsevier: Amsterdam, The Netherlands, 2006.

11. Podlubny, I. Fractional Differential Equations; Acad. Press: Boston, MA, USA, 1999.

12. Yakubovich, S.; Luchko, Y. The Hypergeometric Approach to Integral Transforms and Convolutions; Ser. Mathematics and Its Applications 287; Kluwer Acad. Publ.: Dordrecht, The Netherlands; Boston, MA, USA; London, UK, 1994.

13. Mathai, A.M.; Haubold, H.J. Special Functions for Applied Scientists; Springer: Berlin/Heidelberg, Germany, 2008.

14. Gorenflo, R.; Kilbas, A.; Mainardi, F.; Rogosin, S. Mittag-Leffler Functions, Related Topics and Applications, 2nd ed.; Springer: Berlin/Heidelberg, Germany, 2014. [CrossRef]

15. Erdélyi, A. (Ed.) Higher Transcendental Functions; McGraw Hill: New York, NY, USA, 1953; Volume 1-3.

16. Kilbas, A.A. Fractional calculus of the generalized Wright function. Fract. Calc. Appl. Anal. 2005, 8, 113-126.

17. Haubold, H.J.; Mathai, A.M.; Saxena, R.K. Mittag-Leffler functions and their applications. J. Appl. Math. 2011, 51, 298628. [CrossRef]

18. Rogosin, S. The role of the Mittag-Leffler function in fractional modeling. Mathematics 2015, 3, 368-381. [CrossRef]

19. Paneva-Konovska, J. From Bessel to Multi-Index Mittag-Leffler Functions: Enumerable Families, Series in Them and Convergence; World Scientific Publishing: London, UK, 2016.

20. Kiryakova, V. Multiple Dzrbashjan-Gelfond-Leontiev fractional differintegrals. Recent Adv. Appl. Math. 1996, 96, 281-294.

21. Kiryakova, V. Multiple (multiindex) Mittag-Leffler functions and relations to generalized fractional calculus. J. Comput. Appl. Math. 2000, 118, 241-259. [CrossRef]

22. Kiryakova, V. The multi-index Mittag-Leffler functions as important class of special functions of fractional calculus. Comput. Math. Appl. 2010, 59, 1885-1895. [CrossRef]

23. Kilbas, A.A.; Koroleva, A.A.; Rogosin, S.V. Multi-parametric Mittag-Leffler functions and their extension. Fract. Calc. Appl. Anal. 2013, 16, 378-404. [CrossRef]

24. Kiryakova, V. The special functions of fractional calculus as generalized fractional calculus operators of some basic functions. Comput. Math. Appl. 2010, 59, 1128-1141. [CrossRef]

25. Dzrbashjan, M.M. On the integral transformations generated by the generalized Mittag-Leffler function. Izv. Arm. SSR 1960, 13, 21-63. (In Russian)

26. Paneva-Konovska, J. Multi-index (3m-parametric) Mittag-Leffler functions and fractional calculus. Compt. Rend. Acad. Bulg. Sci. 2011, 64, 1089-1098.

27. Kiryakova, V. A guide to special functions in fractional calculus. Math. Spec. Issue Spec. Funct. Math. Phys. Part II 2020, Submitted.

28. Luchko, Y.; Kiryakova, V. The Mellin integral transform in fractional calculus. Fract. Calc. Appl. Anal. 2013, 16, 405-430. [CrossRef]

29. Agarwal, P.; Rogosin, S.V.; Trujillo, J.J. Certain fractional integral operators and the generalized multi-index Mittag-Leffler functions. Proc. Indian Acad. Sci. (Math. Sci.) 2015, 125, 291-306. [CrossRef]

30. Paneva-Konovska, J.; Kiryakova, V. On the multi-index Mittag-Leffler functions and their Mellin transforms. Int. J. Appl. Math. 2020, 33, 549-571. [CrossRef]

31. Samko, S.; Kilbas, A.; Marichev, O. Fractional Integrals and Derivatives: Theory and Applications; Gordon and Breach: Yverdon, Switzerland, 1993. 
32. Sandev, T.; Tomovski, Ž. Fractional Equations and Models (Theory and Applications); Springer: Berlin/Heidelberg, Germany, 2019. [CrossRef]

33. Sneddon, I.N. The use in mathematical analysis of Erdélyi-Kober operators and some of their applications. In Fractional Calculus and Its Applications (Proc. Internat. Conf. Held in New Haven); Ross, B., Ed.; Lecture Notes in Math. 457; Springer: New York, NY, USA, 1975, pp. 37-79.

34. Kiryakova, V. Generalized fractional calculus operators with special functions. In Handbook of Fractional Calculus with Applications. Volume 1: Basic Theory; Kochubei, A., Luchko, Y., Eds.; De Gryuter: Berlin, Germany, 2019; pp. 87-110. [CrossRef]

35. Kiryakova, V.; Luchko, Y. Riemann-Liouville and Caputo type multiple Erdélyi-Kober operators. Cent. Eur. J. Phys. 2013, 11, 1314-1336. [CrossRef]

36. Luchko, Y.; Trujillo, J.J. Caputo type modification of the Erdélyi-Kober fractional derivative. Fract. Calc. Appl. Anal. 2007, 10, 249-267.

37. Kiryakova, V. A brief story about the operators of the generalized fractional calculus. Fract. Calc. Appl. Anal. 2008, 11, 203-220.

38. Kiryakova, V. Gel'fond-Leont'ev integration operators of fractional (multi-)order generated by some special functions. AIP Conf. Proc. 2018, 2048, 050016. [CrossRef]

39. Marichev, O.I. Volterra equation of Mellin convolutional type with a Horn function in the kernel. Izv. AN BSSR, Ser. Fiz.-Mat. Nauk 1974, 1, 128-129. (In Russian)

40. Saigo, M.; Maeda, N. More generalization of fractional calculus. In Transform Methods E Special Functions, Varna'96 (Proc. Second In- ternat. Workshop); Rusev, P., Dimovski, I., Kiryakova, V., Eds.; Science Culture Technology Publishing: Singapore, 1998; pp. 386-400.

41. Dimovski, I. Operational calculus for a class of differental operators. C.R. Acad. Bulg. Sci. 1966, 19, 1111-1114.

42. Dimovski, I.; Kiryakova, V. Generalized Poisson transmutations and corresponding representations of hyper-Bessel functions. C. R. Acad. Bulg. Sci. 1986, 39, 29-32.

43. Delerue, P. Sur le calcul symboloque à $n$ variables et fonctions hyperbesseliennes (II). Annales Soc. Sci. Bruxelles, Ser. 1 1953, 3, 229-274.

44. Erdélyi, A. (Ed.) Tables of Integral Transforms; McGraw Hill: New York, NY, USA, 1954; Volume 1-2.

45. Askey, R. Orthogonal Polynomials and Special Functions; SIAM: Philadelphia, PA, USA, 1975.

46. Lavoie, J.L.; Osler, T.J.; Tremblay, R. Fractional derivatives and special functions. SIAM Rev. 1976, 18, $240-268$. [CrossRef]

47. Ali, R.S.; Mubeen, S.; Ahmad, M.M. A class of fractional integral operators with multi-index Mittag-Leffler $k$-function and Bessel $k$-function of first kind. J. Math. Comput. Sci. 2020, 22, 266-281. [CrossRef]

48. Mubeen, S.; Ali, R.S.; Nayab, I.; Rahman, G.; Abdeljavad, T.; Nisar, K.S. Integral transforms of an extended generalized multi-index Bessel function. AIMS Math. 2020, 5, 7531-7546. [CrossRef]

49. Abdalla, M. Fractional operators for the Wright hypergeometric matrix functions. Adv. Differ. Equ. 2020, 2020, 246. [CrossRef]

50. Kiryakova, V. All the special functions are fractional differintegrals of elementary functions. J. Phys. A Math. Gen. 1997, 30, 5085-5103. [CrossRef]

51. Mathai, A.M.; Saxena, R.K.; Haubold, H.J. The H-Function; Springer: Berlin/Heidelberg, Germany, 2010.

52. Garrappa, R.; Kaslik, E.; Popolizio, M. Evaluation of fractional integrals and derivatives of elementary functions: Overview and tutorial. Mathematics 2019, 7, 407. [CrossRef]

53. Kilbas, A.A.; Sebastian, N. Generalized fractional integration of Bessel function of first kind. Integr. Transf. Spec. Funct. 2008, 19, 869-883. [CrossRef]

54. Sharma, M.; Jain, R. A note on a generalized $M$-series as a special function of fractional calculus. Fract. Calc. Appl. Anal. 2009, 12, 449-452.

55. Lavault, C. Fractional calculus and generalized Mittag-Leffler type functions. arXiv 2017, arXiv:1703.01912.

56. Kumar D.; Saxena, R.K. Generalized fractional calculus of the $M$-Series involving $F_{3}$ hypergeometric function. Sohag. J. Math. 2015, 2, 17-22. [CrossRef]

57. Saigo, M. A remark on integral operators involving the Gauss hypergeometric functions. Math. Rep. Coll. Gen. Ed. Kyushu Univ. 1978, 11, 135-143.

58. Kiryakova, V. On two Saigo's fractional integral operators in the class of univalent functions. Fract. Calc. Appl. Anal. 2006, 9, 159-176. 
59. Sharma, K. An introduction to the generalized fractional integration. Bol. Soc. Paran. Math. 2012, 30, 85-90. [CrossRef]

60. Purohit, S.D.; Suthar, D.L.; Kalla, S.L. Marichev-Saigo-Maeda fractional integration operators of the Bessel functions. Le Mat. 2012, LXVII, 21-32. [CrossRef]

61. Agarwal, R.; Jain, S.; Agarwal, R.P.; Baleanu, D. Response: Commentary: A remark on the fractional integral operators and the image formulas of generalized Lommel-Wright function. Front. Phys. 2020, 8, 72. [CrossRef]

62. Agarwal, R.; Jain, S.; Agarwal, R.P.; Baleanu, D. A remark on the fractional integral operators and the image formulas of generalized Lommel-Wright function. Front. Phys. 2018, 6, 79. [CrossRef]

63. Mondal, S.R.; Nisar, K.S. Marichev-Saigo-Maeda fractional integration operators involving generalized Bessel functions. Math. Probl. Eng. 2014, 11, 274093. [CrossRef]

64. Nisar, K.S.; Mondal, S.R.; Agarwal, P. Composition formulas of Bessel-Struve kernel function. arXiv 2016, arXiv:1602.00279v1.

65. Srivastava, H.M.; Tomovski, Ž. Fractional calculus with an integral operator containing generalized Mittag-Leffler function in the kernel. Appl. Math. Comput. 2009, 211, 198-210. [CrossRef]

66. Saxena, R.K.; Nishimoto, K. N-fractional calculus of generalized Mittag-Leffler functions. J. Fract. Calc. 2010, $37,43-52$.

67. Saxena, R.K.; Pogany, T.K.; Ram, J.; Daiya, J. Dirichlet averages of generalized multi-index Mittag-Leffler functions. Armen. J. Math. 2010, 3, 174-187.

68. Kumar, D.; Kumar Gupta, R.; Singh Rawat, D. Marichev-Saigo-Maeda fractional differential operator involving mittag-Leffler type function with four parameters. J. Chem. Biol. Phys. Sci. Sect. C: Phys. Sci. 2017, 7, 201-210.

69. Kiryakova, V. From the hyper-Bessel operators of Dimovski to the generalized fractional calculus. Fract. Calc. Appl. Anal. 2014, 17, 977-1000. [CrossRef]

70. Gehlot, K.S. Differential equation of $k$-Bessel's function and its properties. Nonl. Anal. Differ. Equ. 2014, 2, 61-67. [CrossRef]

71. Mondal, S.R. Representation formulae and monotonicity of the generalized $k$-Bessel functions. arXiv 2016, arXiv:1611.07499.

72. Shaktawat, B.S.; Rawat, D.S.; Gupta, R.K. On generalized fractional calculus of the generalized k-Bessel function. J. Rajasthan Acad. Phys. Sci. 2017, 16, 9-19.

73. Dorrego, G.A.; Cerruti, R.A. The k-Mittag-Leffler function. Int. J. Contemp. Math. Sci. 2012, 7, 705-716.

74. Gupta, A.; Parihar, C.L. k-New generalized Mittag-Leffler function. J. Fract. Calc. Appl. 2014, 5, $165-176$.

75. Nisar, K.S.; Eata, A.F.; Al-Dhaifallah, M.; Choi, J. Fractional calculus of generalized $k$-Mittag-Leffler function and its applications to statistical distribution. Adv. Differ. Equ. 2016, 304. [CrossRef]

76. Nisar, K.S.; Purohit, S.D.; Parmar, R.K. Fractional calculus and certain integrals of generalized multiindex Bessel function. arXiv 2017, arXiv:1706.08039.

77. Saxena, R.K.; Daiya, J. Integral transforms of S-functions. Le Mat. 2015, LXX, 147-159.

78. Purohit, M.; Badguzer, A. MSM fractional integration and differentiation operators of multi-parametric K-Mittag Leffler function and generalized multi-index Bessel function. Intern. J. Stat. Appl. Math. 2018, 3, $156-161$.

Publisher's Note: MDPI stays neutral with regard to jurisdictional claims in published maps and institutional affiliations.

(C) 2020 by the author. Licensee MDPI, Basel, Switzerland. This article is an open access article distributed under the terms and conditions of the Creative Commons Attribution (CC BY) license (http:/ / creativecommons.org/licenses/by/4.0/). 